\title{
LOCOMOTION, WRINKLING, AND BUDDING OF A MULTICOMPONENT VESICLE IN VISCOUS FLUIDS*
}

\author{
SHUWANG LI $^{\dagger}$, JOHN LOWENGRUB ${ }^{\ddagger}$, AND AXEL VOIGT ${ }^{\S}$
}

\begin{abstract}
Recent experimental results on giant unilamellar vesicles (GUVs) show that mixed multiple lipid components on the surface of a membrane may decompose into coexisting phases with distinct compositions, with concomitant changes in the surface morphology. The driving forces for the evolution involves bending, line tension along the phase boundaries, inhomogeneous surface energy, and fluid forces. Here we are interested in exploring the emergent morphologies when the flow is present, and in particular when the surface tensions of the coexisting phases are different, which has not been considered previously. In this paper, we present a model capable of describing the nonlinear coupling among flow, membrane morphology, and the evolution of the surface phases. Using an energy variation approach, we derive a generalized surface tension and construct a constitutive equation connecting the surface tension and the phase variables. To investigate the nonlinear dynamics, we develop a numerical method that combines the immersed interface method to solve the flow equations, the level-set method to capture the interface motion, a non-stiff Eulerian algorithm to solve the phase field equations on the evolving surface, and a penalty term that enforces global inextensibility. Our numerical results suggest that the nonhomogeneous surface tension, together with the flow, introduces nontrivial vesicle dynamics including locomotion, wrinkling, and budding.
\end{abstract}

Key words. Multicomponent vesicle, ordered and disordered lipid phases, line tension, inextensibility, Stokes flow.

AMS subject classifications. 35R35, 35R37, 76D45, 92B05.

\section{Introduction}

Membranes are composed of bilayer lipid molecules with a hydrophilic heads and two hydrophobic hydrocarbon chains. In an aqueous environment, membranes form vesicles - encapsulating bag-like shapes to reduce the energy of the hydrophobic edges. As the principal components of living organisms, membranes contain a mixture of materials including lipids, proteins, and cholesterols that may decompose into coexisting domains of different phases $[1,2,3]$. To facilitate their biological functions such as solute and chemical transport [4], membranes interact with their fluid surroundings in surprising ways and exhibit rich shape transition behaviors [5, 6]. At the continuum level, the mathematical description of morphological changes due to these coupled physical phenomena poses a highly challenging nonlinear moving boundary problem. In this paper, we develop a thermodynamically consistent model for investigating the effects of flow and a generalized surface tension on membrane morphologies.

Studies of the membrane morphologies arise from trying to understand the underlying biophysics. From a mathematical point of view, however, studies of the membrane morphologies have led to many interesting and fundamental problems such as minimization of energy functionals of the membrane system. Julicher and Lipowsky developed a theory on the equilibrium shapes of two-component vesicles without the

${ }^{*}$ Received: December 17, 2010; accepted (in revised version): August 23, 2011. Communicated by Peter Smereka.

${ }^{\dagger}$ Department of Applied Mathematics, Illinois Institute of Technology, Chicago, IL 60616, USA (sli@math.iit.edu).

${ }^{\ddagger}$ Department of Mathematics, University of California, Irvine, CA 92697, USA (lowengrb@math.uci.edu).

§Institut fur Wissenschaftliches rechnen, Technische Universität Dresden, Zellescher Weg 12-14, 01062 Dresden, Germany (Axel.Voigt@tu-dresden.de). 
presence of flow [7]. Using Lagrange multipliers to enforce constraints of surface area (i.e. no free lipid molecules in aqueous solution) and volume (i.e. no osmotic pressure building up inside the vesicle), they obtained the Euler-Lagrange equations for the equilibrium shape and investigated the competition between the bending force and the line tension along the phase boundaries. Their results show that vesicles containing an intramembrane domain may form a bud (i.e. growth of a small vesicle from a larger one) provided the domain size is large enough. This theoretical study was later successfully used by Baumgart et al. to compare with their experimental results on giant unilamellar vesicles (GUV) [5].

The morphology of an evolving membrane is the result of the nonlinear interactions among bending, line tension, inhomogeneous surface energy, fluid forces, and phase transitions on the membrane surface. A number of models and associated numerical algorithms have been developed for investigating the effects of these physical processes on membrane morphologies; see the reviews $[8,2,1,9,10]$, and the references therein.

Bending elasticity has been studied extensively in vesicle dynamics because of membrane's highly flexible bi-layer thin structures. In the absence of fluid flow, Taniguchi developed a dynamical approach that couples interface dynamics with a surface phase-field equation [11]. This model suggests that the local coupling of the curvature and the composition of the membrane and the line tension at domain boundaries strongly influence the formation of protrusions (buds) on the membrane, which may undergo fission at a later stage, i.e. separation of a bud from its mother membrane. Wang and Du formulated a phase field model for multicomponent vesicles with free edges and simulated exotic patterns observed in experiments [12]. Boundary integral methods have also been successfully used to simulate the vesicle dynamics in a viscous fluid $[13,14,15,16]$, such as tank-treading and tumbling [16].

In addition, vesicle wrinkling has been observed in experiments of elastic capsules in an applied shear flow by Walter et al. [17]. Finken and Seifert [18] performed a mathematical analysis and derived analytical results both for the threshold value of the shear rate and for the critical wavelength of the wrinkling. Kantsler et al. also found that vesicle wrinkling may occur in time-dependent elongation flow $[19,6]$. It has been suggested that the winkling phenomena are related to the dynamical instability induced by negative surface tension of the membrane [20].

Further, it has been demonstrated in experiments that surface tension can also lead to nontrivial shapes by external means [21]. Bar-Ziv et al. [22] reported that when optical tweezers are used to perturb a biomembrane for a few minutes, the membrane shape stops fluctuating and becomes very tense, indicating the significance of surface tension; the surface tension can be as large as $10^{-3} \mathrm{erg} / \mathrm{cm}^{2}$ or greater; see also [23]. Moreover, this strong surface tension is involved in the opening of a hole inside the membrane and results in the expulsion of a bud-like vesicle from inside of the mother vesicle [22]. Without the stimulation of laser tweezers, surface tension, though small, is found to be on the order of $\sigma \approx 10^{-6} \mathrm{erg} / \mathrm{cm}^{2}$ by mechanical manipulations [23]. The surface tension considered in these experiments is an effective tension that arises due to inextensibility. That is, the interface cannot stretch globally or locally. The concept of effective tension was developed by Seifert for fluctuating vesicles in [24], in which he demonstrated that the area constraint works like an effective tension whose value is calculated by a Lagrange multiplier used to determine the mean shape. So the surface tension only represents the area constraint in a conjugated sense.

Surface tension considered in this paper is coupled with the phase decomposition 
of the nonhomogeneous mixture (e.g. cholesterol and lipids) on the membrane surface. Experimental results show that an unstable mixture may decompose into phases with distinct compositions. For example, recent results of giant unilamellar vesicles (GUVs) reveal that membranes initially containing ternary mixtures of lipid components and cholesterol may separate into binary ordered/disordered liquid phases (i.e. coexistence of distinct fluid bilayer domains on the membrane surface) $[25,5,26]$. Therefore, from the point view of surface phases, it is reasonable to expect that the surface energy may depend on the local composition, and thus may play a role in the vesicle dynamics via the coupling with surrounding flow around the vesicle. We note that in order to explore this possibility, one needs to derive a generalized surface tension and a constitutive equation connecting the surface tension and phase compositions.

In this paper, using a simplified version of the Helfrich model for fluid-like vesicle membranes [27], we develop a thermodynamically consistent model of a multicomponent membrane. To isolate the effects of surface tension on the membrane morphological transitions, we neglect the bending forces and spontaneous curvature. We assume the energy of the system, which serves as the driving force for dynamical changes, comes from two sources: surface energy of the vesicle that depends on the concentration of the surface components (e.g. phase-field variables); and chemical energy of the surface phases that is taken to be of Ginzburg-Landau form. Using an energy variation approach, we derive the thermodynamically consistent constitutive equations for: (1) the generalized surface tension forces imparted to the flow as a boundary condition at the membrane surface; (2) the diffusion flux imparted to the mass conservation equations of Cahn-Hilliard type on the evolving membrane surface. These two constitutive relations guarantee that the free energy of the system is non-increasing or equivalently for isothermal systems that the entropy is non-decreasing. The surface phases evolve according to a high-order, advection-reaction-diffusion equation of Cahn-Hilliard type on the moving surface. A penalty formulation is used to enforce global inextensibility as in [28]. This simplified model is capable of describing the nonlinear coupling among the flow, vesicle morphology and the evolution of the surface phases. It serves as preparatory for a more complete study of multicomponent vesicles.

Recently, Sohn et al. [16] considered multicomponent vesicle dynamics with inhomogeneous bending stiffnesses in 2D. Local inextensiblity was enforced using a Lagrange multiplier, which induces a tension along the interface. The boundary integral approach that was used in this work naturally extends to axisymmetric geometries but is hard to extend to full 3D vesicles. Moreover, Sohn et al. did not observe wrinkling instabilities.

The simplified model investigated here provides a framework that can be more easily extended to simulate $3 \mathrm{D}$ vesicle morphologies. However, there are hurdles that must be overcome to achieve this goal, including the development of accurate discretization techniques for the bending forces and adaptive mesh refinement techniques to improve efficiency and accuracy. Both of these techniques are needed to accurately simulate the dynamics of vesicles, with inhomogeneous bending forces, immersed in a fluid. This is currently under development and here we focus on a proof-of-principle approach that accounts for inhomogeneous surface energies, inextensibility, and fluid flow, and demonstrates the potential of this modeling approach to simulate the locomotion, wrinkling, and budding of multicomponent vesicles.

To solve the highly nonlinear coupled system, we develop an efficient nonlinear numerical method based on the work developed in [29]. The method combines the immersed interface method to solve the flow equations with generalized Laplace-Young 
jump conditions for the jump in normal stress of the fluid across the membrane, the level-set method to capture and evolve the interface and a non-stiff Eulerian algorithm to update the mass concentration on the membrane surface [29]. While there has been recent progress on modeling local inextensibility conditions for implicitly defined interfaces (e.g., [30, 31]), the problem is still not solved and work is on-going to complete the local inextensibility description. Consequently, here we use a penalty approach to enforce this constraint.

Our numerical results on the dynamics of two-dimensional vesicles reveal that nonhomogeneous surface tension, coupled with the surrounding viscous fluid, introduces vesicle locomotion and budding. When the arclength constraint is not present, an initially perturbed circular vesicle will evolve back to a circular shape with wellseparated phases. Because of the unbalanced surface tension between two phases, vesicles move towards the direction in which surface tension is small. When the arclength constraint is enforced, in addition to self-locomotion, a perturbed circular vesicle exhibits various morphologies during evolution. Examples include the formation of wrinkles for vesicles with limited excess arclength and buds for vesicles with larger excess arclength.

The paper is organized as follows. In Section 2, we derive and nondimensionalize the governing equations. In Section 3, we describe the numerical algorithms. In Section 4, we present preliminary numerical results. In Section 5, we give concluding remarks and discuss potential future work.

\section{Formulation}

\subsection{Governing equations.}

2.1.1. Flow field. Consider a vesicle containing one viscous fluid inside and surrounded by another viscous fluid outside. Let $\Sigma$ denote the interface separating the fluids. We assume that both fluids are highly viscous and satisfy the Stokes equations,

$$
\nabla \cdot \mathbf{T}_{i}=0 \quad \text { and } \quad \nabla \cdot \mathbf{u}_{\mathrm{i}}=0 \quad \text { in } \quad \Omega_{\mathrm{i}},
$$

where $\mathbf{T}_{i}=-p_{i} I+\eta_{i}\left(\nabla \mathbf{u}_{i}+\nabla \mathbf{u}_{i}^{T}\right)$ is the viscous stress tensor, $p_{i}$ is the pressure, and $i=1,2$ denotes the interior vesicle and exterior matrix fluid regions respectively.

The velocity is continuous across the interface $\Sigma$,

$$
[\mathbf{u}]_{\Sigma} \equiv\left(\left.\mathbf{u}\right|_{\Sigma, 2}-\left.\mathbf{u}\right|_{\Sigma, 1}\right)=0
$$

and the stress has a jump across the interface given by Laplace-Young jump condition

$$
[\mathbf{T n}]_{\Sigma}=\sigma \kappa \mathbf{n}-\nabla_{s} \sigma
$$

where $\sigma$ is the surface tension whose constitutive relation will be derived below in Equation(2.21), $\mathbf{n}$ is the outward normal vector to $\Sigma(t)$ pointing into the matrix fluid, $\kappa=\nabla \cdot \mathbf{n}$ is the curvature of $\Sigma$ (positive for convex interface), and $\nabla_{s}$ is the surface gradient. At the far-field, we supply a flow

$$
\mathbf{u}=\mathbf{u}_{\infty} \quad \text { on } \quad \partial \Omega
$$

where $\Omega=\Omega_{1} \cup \Omega_{2}$. Finally, the interface $\Sigma(t)=\left\{\mathbf{x}_{\Sigma}(t)\right\}$ moves with the fluid with velocity

$$
\frac{d \mathbf{x}_{\Sigma}}{d t}=\mathbf{u}
$$


2.1.2. Material field. For simplicity, we focus our study on two surface phases (e.g. lipid components). Let $f(s, t)$ denote the mass concentration of one surface phase; the concentration of the other phase is $\bar{f}(s, t)-f(s, t)$, where $\bar{f}$ is the total concentration of the surface phases and $s$ parameterizes the interface $\Sigma$. Here, $\bar{f}(s, t)$ and $f(s, t)$ are functions of time and space. We assume that no reactions occur and that the phases are distributed only on the interface. Therefore, the total mass is conserved and mass of both surface phases are conserved. Consequently, $f(s, t)$ satisfies

$$
M_{f}(t)=\int_{\Sigma(t)} f(s, t) d \Sigma=M_{f}(0),
$$

where we have implicitly assumed, for simplicity, that the surface density of each phase is equal to one. Accordingly, the surface concentration $f(s, t)$ evolves via a convection-reaction-diffusion equation. In Eulerian coordinates, it reads

$$
f_{t}+\mathbf{u} \cdot \nabla f-\mathbf{n} \cdot \nabla \mathbf{u} \cdot \mathbf{n} f=\nabla_{s} \cdot \mathbf{J}_{s},
$$

where $\mathbf{J}_{s}$ is the surface flux whose constitutive relation will be derived below in Equation (2.20), and the expression

$$
-\mathbf{n} \cdot \nabla \mathbf{u} \cdot \mathbf{n}=\nabla_{s} \cdot \mathbf{u}_{s}+\kappa \mathbf{u} \cdot \mathbf{n},
$$

where $\mathbf{u}_{s}$ is the tangential velocity on $\Sigma$. For an incompressible velocity field, the left hand side of Equation (2.8) describes the local rate of change of the interface area. Correspondingly, this term in Equation (2.7) describes the change in $f$ due to interface stretching [32, 33, 29].

To conserve the total mass, we have

$$
M_{t o t}(t)=\int_{\Sigma(t)} \bar{f}(s, t) d \Sigma=M_{t o t}(0) .
$$

The local version of the total mass conservation equation reads

$$
\bar{f}_{t}+\mathbf{u} \cdot \nabla \bar{f}-\mathbf{n} \cdot \nabla \mathbf{u} \cdot \mathbf{n} \bar{f}=0 .
$$

2.2. Constitutive relations. To solve the flow and phase fields, we need to specify constitutive relations for the surface tension $\sigma$ and the diffusion flux $\mathbf{J}_{s}$. This is done by an energy variation approach so that the resulting system is consistent with the second law of thermodynamics and couples the surface phase separation with the fluid mechanics. To satisfy the second law of thermodynamics, it is sufficient to obtain a non-increasing free energy functional since this is equivalent to a nondecreasing entropy (e.g., the Helmholtz free energy $H=e-T S$ where $e$ is the internal energy, $T$ is the temperature, and $S$ is the entropy; e.g. see [34]). We consider the free energy of the system

$$
\begin{aligned}
E & =E_{s}+E_{f}+E_{p}, \quad \text { with } \\
E_{s} & =\int_{\Sigma(t)} \gamma(\bar{f}, f) d \Sigma, \\
E_{f} & =\int_{\Sigma(t)}\left(g(\bar{f}, f)+\frac{\epsilon^{2}}{2}\left|\nabla_{s} f\right|^{2}\right) d \Sigma,
\end{aligned}
$$




$$
\text { and } \quad E_{p}=\frac{\lambda}{2}\left(\int_{\Sigma(t)} d \Sigma-\int_{\Sigma(0)} d \Sigma\right)^{2}
$$

where $E_{s}$ is the surface energy, $E_{f}$ is a generalized chemical free energy associated with the surface phases, and $E_{p}$ is a penalty term to impose the area constraint. In Equation (2.12), the function $\gamma(\bar{f}, f)$ is the surface energy density of the surface phase components. In Equation (2.13), the function $g(\bar{f}, f)$ is the chemical free energy (e.g. double-well potential for a two-phase system) and $\epsilon$ is a parameter (taken to be constant for simplicity) that measures the excess energy due to surface gradients. Under appropriate scaling conditions, $E_{f}$ represents the line-energy separating the surface domains. In Equation (2.14), we enforce the area constraint in a global sense, i.e. we want the total surface area at time $t, \int_{\Sigma(t)} d \Sigma=\int_{\Sigma(0)} d \Sigma=A(0)$ where $A(0)$ is the desired area, and any deviation in the area $A(t)$ from the desired area $A(0)$ is associated with high energy configurations and is not favored by the system. The parameter $\lambda$ is used to measure the contribution of the penalty term in total energy. In a recent boundary integral formulation [16], we enforce a local surface area constraint using a Lagrange multiplier method.

Next, we take the time derivative of Equation (2.11). That is, we vary both the interface $\Sigma(t)$ and the concentration function $f(t, s)$ simultaneously and independently. A calculation in Eulerian coordinates yields

$$
\dot{E}_{s}=\int_{\Sigma(t)}\left(\frac{\partial \gamma}{\partial \bar{f}} \dot{\bar{f}}+\frac{\partial \gamma}{\partial f} \dot{f}-\gamma \mathbf{n} \cdot \nabla \mathbf{u} \cdot \mathbf{n}\right) d \Sigma
$$

where $\dot{f}=\partial f / \partial t+\mathbf{u} \cdot \nabla f$ and $\dot{\bar{f}}$ is defined analogously. Note that in defining $\dot{f}$, we have implicitly assumed that $f$ is extended off the interface such that $\nabla f \cdot \mathbf{n}=0$ in a small neighborhood of $\Sigma$; thus $\nabla f=\nabla_{s} f$ (likewise for $\dot{\bar{f}}$ if $\bar{f}$ is space dependent). A similar calculation shows that

$$
\begin{gathered}
\dot{E}_{f}=\int_{\Sigma(t)}\left(\dot{\bar{f}} \frac{\partial g}{\partial \bar{f}}+\dot{f}\left(\frac{\partial g}{\partial f}-\epsilon^{2} \Delta_{s} f\right)-\epsilon^{2} \nabla_{s} f \cdot \nabla \mathbf{u} \cdot \nabla_{s} f\right) d \Sigma \\
+\int_{\Sigma(t)}\left(g(f)+\frac{\epsilon^{2}}{2}\left|\nabla_{s} f\right|^{2}\right)(\mathbf{I}-\mathbf{n n}): \nabla \mathbf{u} d \Sigma
\end{gathered}
$$

and

$$
\dot{E}_{p}=\lambda \int_{\Sigma(t)}(\mathbf{I}-\mathbf{n n}): \nabla \mathbf{u} d \Sigma\left(\int_{\Sigma(t)} d \Sigma-\int_{\Sigma(0)} d \Sigma\right),
$$

where $\Delta_{s}=\nabla_{s} \cdot \nabla_{s}$ is the Laplace-Beltrami operator (surface Laplacian) and the notation $\mathbf{a}: \mathbf{b}=\sum_{i j} a_{i j} b_{i j}$ denotes the tensor product. In deriving equations (2.16) and (2.17), we have used integration by parts and the incompressibility of the velocity field $\mathbf{u}$.

For simplicity, we focus on the two-dimensional case in the remainder of this paper, so surface area reduces to the arclength of the membrane, $L(t)$ at time $t$; the $3 \mathrm{D}$ problem will be considered in a future work. In $2 \mathrm{D}, \nabla_{s} f \cdot \nabla \mathbf{u} \cdot \nabla_{s} f=\left|\nabla_{s} f\right|^{2}(\mathbf{I}-\mathbf{n n})$ : $\nabla \mathbf{u}$. In 3D, this becomes $\nabla_{s} f \nabla_{s} f: \nabla \mathbf{u}$. 
Putting this together with equations (2.7), (2.10), (2.15), (2.16), and (2.17), and integrating by parts, we get

$$
\dot{E}=\int_{\Sigma(t)}\left(\nabla_{s} \cdot \mathbf{J}_{s}\right) \mu d \Sigma+\int_{\Sigma(t)} \mathbf{u} \cdot\left(-\nabla_{s} \sigma+\kappa \sigma \mathbf{n}\right) d \Sigma,
$$

where $\mu$ is the chemical potential defined by

$$
\mu=\frac{\partial g}{\partial f}-\epsilon^{2} \Delta_{s} f+\frac{\partial \gamma}{\partial f}
$$

The constitutive relation for the surface flux thus can be defined by

$$
J_{s}=\nu \nabla_{s} \mu,
$$

where $\nu$ is the mobility. Note that Equation (2.20) takes the form of a generalized Fick's law. In the second integral of Equation (2.18), the constitutive relation for a generalized surface tension $\sigma$ is defined as

$$
\begin{array}{r}
\sigma(f)=g(\bar{f}, f)-f \frac{\partial g}{\partial f}-\bar{f} \frac{\partial g}{\partial \bar{f}}-\frac{\epsilon^{2}}{2}\left|\nabla_{s} f\right|^{2}+\epsilon^{2} f \Delta_{s} f \\
+\tau(\bar{f}, f)+\lambda(L(t)-L(0)),
\end{array}
$$

where $\tau$ is the surface tension of the surface phase components in the absence of phase separation,

$$
\tau(\bar{f}, f)=\gamma(\bar{f}, f)-f \frac{\partial \gamma}{\partial f}-\bar{f} \frac{\partial \gamma}{\partial \bar{f}}
$$

In constructing the constitutive relations for $\mathbf{J}_{s}$ and $\sigma$, we assume chemical and viscous dissipations are not coupled.

If there is no energy added into the system (i.e. $\mathbf{u}_{\infty}=0$ ), we substitute the constitutive relations equations (2.20), (2.21) and the stress jump condition (2.3) into Equation (2.18), perform integration by parts using the divergence theorem, and get that the total energy of the system is decreasing in time,

$$
\begin{gathered}
\dot{E}=-\int_{\Sigma(t)} \nu\left|\nabla_{s} \mu\right|^{2} d \Sigma-\frac{1}{2} \int_{\Omega_{1}} \eta_{1}\left(\nabla \mathbf{u}_{1}+\nabla \mathbf{u}_{1}^{T}\right):\left(\nabla \mathbf{u}_{1}+\nabla \mathbf{u}_{1}^{T}\right) d \mathbf{x} \\
-\frac{1}{2} \int_{\Omega_{2}} \eta_{2}\left(\nabla \mathbf{u}_{2}+\nabla \mathbf{u}_{2}^{T}\right):\left(\nabla \mathbf{u}_{2}+\nabla \mathbf{u}_{2}^{T}\right) d \mathbf{x} .
\end{gathered}
$$

Thus, the constitutive assumptions in equations (2.20) and (2.21) are consistent with the second law of thermodynamics.

2.3. Nondimensionalization. Let the vesicle radius, $a$, be the length scale, $a / \bar{U}$ be the time scale, where $\bar{U}$ is a characteristic velocity, $\bar{p}=\eta_{2} \bar{U} / a$ be the characteristic stress, and $\bar{\sigma}$ be a characteristic surface tension. Denoting the nondimensional quantities, i.e. $\tilde{\mathbf{x}}=\mathbf{x} / a, \tilde{\mathbf{T}}_{i}=\mathbf{T}_{i} / \bar{p}, \tilde{\mathbf{u}}_{i}=\mathbf{u}_{i} / \bar{U}, \tilde{\sigma}=\sigma / \bar{\sigma}$ etc., we obtain the nondimensional Stokes equations after dropping the tilde notation,

$$
\nabla \cdot \mathbf{T}_{i}=0, \quad \nabla \cdot \mathbf{u}_{i}=0, \quad \text { for } \quad i=1,2,
$$

where stress tensor $\mathbf{T}_{i}=-p_{i} \mathbf{I}+\lambda_{i}\left(\nabla \mathbf{u}_{i}+\nabla \mathbf{u}_{i}^{T}\right)$ and the viscosity ratio $\lambda_{2}=1$ and $\lambda_{1}=\eta_{1} / \eta_{2}$. 
The velocity is continuous across $\Sigma$ (e.g. Equation (2.2) is satisfied for $\mathbf{u}_{i}$ ) and the Laplace-Young condition (2.3) becomes

$$
[\mathbf{T n}]_{\Sigma}=\frac{1}{C a}\left(\sigma \kappa \mathbf{n}-\nabla_{s} \sigma\right),
$$

where $C a=\eta_{2} \bar{U} / \bar{\sigma}$ is the Capillary number. Letting $\bar{\mu}$ be the characteristic chemical energy scale, we obtain from Equation (2.21) the nondimensional surface tension

$$
\begin{array}{r}
\sigma=\frac{1}{\mathcal{M}}\left(g(\bar{f}, f)-f \frac{\partial g}{\partial f}-\bar{f} \frac{\partial g}{\partial \bar{f}}-\frac{\mathcal{C}^{2}}{2}\left|\nabla_{s} f\right|^{2}+\mathcal{C}^{2} f \Delta_{s} f\right) \\
+\tau(\bar{f}, f)+\Lambda(L(t)-L(0))
\end{array}
$$

where $f$ is already nondimensional, $g$ is the nondimensional chemical free energy obtained by scaling the dimensional form by $\bar{\mu}$, and $\tau$ is the nondimensional surface tension obtained by scaling the dimensional form by $\bar{\sigma}, \mathcal{C}=\epsilon /(a \sqrt{\bar{\mu}})$ is the Cahn number, $\Lambda=\lambda a / \bar{\sigma}$, and $\mathcal{M}=\bar{\sigma} / \bar{\mu}$, which works like the Mach number [35] and measures the relative strengths of the surface tension and chemical forces. Accordingly, the nondimensional chemical potential is given by

$$
\mu=\frac{\partial g}{\partial f}-\mathcal{C}^{2} \Delta_{s} f+\mathcal{M} \frac{\partial \gamma}{\partial f} .
$$

The nondimensional version of the surface mass transport equations (2.7) and (2.9) are

$$
\begin{aligned}
& f_{t}+\mathbf{u} \cdot \nabla f-\mathbf{n} \cdot \nabla \mathbf{u} \cdot \mathbf{n} f=\frac{1}{P e} \nabla_{s}\left(\nu \nabla_{s} \mu\right), \\
& \bar{f}_{t}+\mathbf{u} \cdot \nabla \bar{f}-\mathbf{n} \cdot \nabla \mathbf{u} \cdot \mathbf{n} \bar{f}=0,
\end{aligned}
$$

where the Peclet number $P e=\bar{\nu} \bar{\mu} /(a \bar{U})$.

\section{Numerical methods}

We use the immersed interface method (IIM) to solve the 2D Stokes equations for the fluid velocity field $\mathbf{u}$. We then use $\mathbf{u}$ to update the position of the interface $\Sigma(t)$ by a level-set method. Once the interface is identified, we use a non-stiff Eulerian algorithm to solve the phase field equations for the concentration $f(t, s)$ on the interface; see Figure 3.1 for a schematic diagram of the algorithm. The algorithm presented below is an extension of the approach developed in [29] for interfacial flows with surfactants. For completeness, we outline the main ideas here. In this paper, we consider the case of viscosity matched fluids $\lambda_{1}=\lambda_{2}=1$. The IIM can also be extended to solve the case with mismatched viscosity by using an augmented variable approach [36].

3.1. The evolution of the mass concentration. For a given initial shape, we first solve the material field and compute the generalized surface tension in Equation (2.26), which will be imparted to the boundary condition for the flow equation. The mass concentration $f$ is evolved in three steps. First, $f$ and the chemical potential $\mu$ are extended off the interface $\Sigma$ into a small tube around $\Sigma$. Second, the surface mass transport Equation (2.28) is solved in the tube using an efficient nonstiff method. Third, the total mass conservation Equation (2.29) is solved using a semi-implicit method. For simplicity, we assume that the mobility $\nu=1$. 


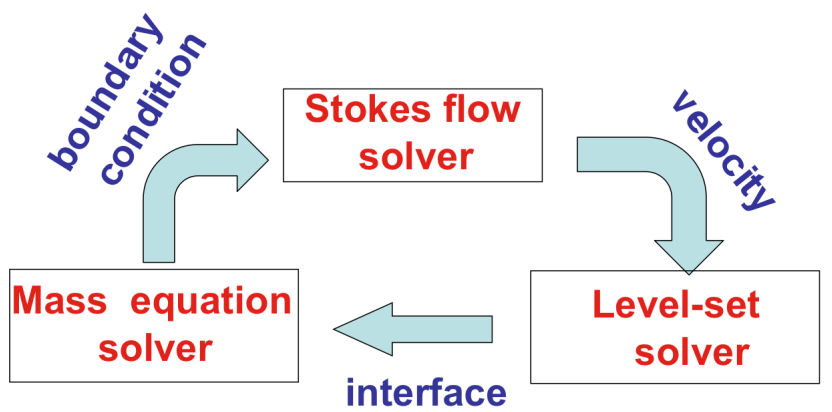

FIG. 3.1. A schematic diagram of the combined numerical method.

3.1.1. Extension of the mass concentration off the interface. Following [37], $f$ is extended off $\Sigma$ by solving the following Hamilton-Jacobian equation:

$$
\left\{\begin{array}{l}
f_{\tau}+S(\phi) \mathbf{n} \cdot \nabla f=0 \\
f(\mathbf{x}, 0)=f_{0}(\mathbf{x})
\end{array}\right.
$$

where as before $S(x)$ is the sign function of $x$ defined below in Equation (3.20) and $\tau$ is a pseudo-time that measures the magnitude of the radius of the tube into which $f$ is extended. Similarly, the chemical potential $\mu$ is extended off $\Sigma$ analogously. Equation (3.1) is solved using the third order WENO and Runge-Kutta spatial and temporal discretizations, respectively.

\subsubsection{A nonstiff method for the surface mass transport equation.}

The surface mass transport equation (2.28) is a nonlinear, fourth-order differential equation. Explicit methods suffer severe time step restrictions for stability (time step $p \sim h^{4}$, where $h$ is the spatial resolution). Here, we use a non-stiff semi-implicit numerical method to remove the stiffness, i.e. we integrate the highest order terms implicitly in time.

We first decompose the Laplace-Beltrami operator as in [38]:

$$
\Delta_{s} \mu=\Delta \mu-\frac{\partial^{2} \mu}{\partial n^{2}}-\kappa \frac{\partial \mu}{\partial n}
$$

where $\Delta=\partial^{2} / \partial x^{2}+\partial^{2} / \partial y^{2}$ is the usual Laplacian. We then decompose the Helmholtz free energy [39]

$$
\frac{\partial g}{\partial f}=\bar{a} f+\left(\frac{\partial g}{\partial f}-\bar{a} f\right)
$$

where $\bar{a}$ is a nonnegative constant ( $\bar{a}$ is typically taken to be max $\left.\left|\frac{\partial^{2} g}{\partial f^{2}}\right|\right)$. We then rewrite Equation (2.28) into a system of two second order differential equations with $f$ and $\mu$ as unknowns,

$$
\begin{aligned}
f_{t}-\frac{1}{P e} \Delta \mu & =F(\mathbf{x}, t), \\
\mu-\bar{a} f+\mathcal{C}^{2} \Delta f & =G(\mathbf{x}, t),
\end{aligned}
$$


where the right hand side is

$$
\begin{aligned}
& F(\mathbf{x}, t)=-\mathbf{u} \cdot \nabla f+\mathbf{n} \cdot \nabla \mathbf{u} \cdot \mathbf{n} f-\frac{1}{P e}\left(\frac{\partial^{2} \mu}{\partial n^{2}}+\kappa \frac{\partial \mu}{\partial n}\right), \\
& G(\mathbf{x}, t)=\frac{\partial g}{\partial f}-\bar{a} f+\frac{\partial \gamma}{\partial f}+\mathcal{C}^{2}\left(\frac{\partial^{2} f}{\partial n^{2}}+\kappa \frac{\partial f}{\partial n}\right) .
\end{aligned}
$$

In practice, the left hand sides of equations (3.4)-(3.5) are discretized implicitly, and the right hand sides are discretized explicitly. In equations (3.4) and (3.5), we use a semi-implicit backward Euler discretization in time to approximate $f_{t}$. The advection term $\mathbf{u} \cdot \nabla f$ is discretized using a third order WENO algorithm, and the other differential terms are discretized using centered differences in space. The resulting linear system is solved in a band around the interface by using an iterative Gauss-Seidel method for $f$ and $\mu$ simultaneously. Using an error tolerance of $10^{-6}$, less than 50 iterations per time step are required. Because the number of updated points scales like $N$, where $N$ is the number of grid points in a one direction, this algorithm is still inexpensive. However, other more efficient solution methods (e.g. multigrid) are under development.

\subsubsection{A semi-implicit method for the total mass transport equation.}

The total mass transport Equation (2.29) is solved using a semi-implicit method [40]. We first rewrite Equation (2.29) as

$$
\overline{f_{t}}=-\beta \Delta^{2} \bar{f}+\beta \Delta^{2} \bar{f}-S(\bar{f}),
$$

where $S(\bar{f})=\mathbf{u} \cdot \nabla \bar{f}-\mathbf{n} \cdot \nabla \mathbf{u} \cdot \mathbf{n} \bar{f}$, and $\beta$ is a constant to be determined. We discretize Equation (3.8) in time (first order accurate) as,

$$
\bar{f}^{n+1}=\bar{f}^{n}-\Delta t \beta \Delta^{2} \bar{f}^{n+1}+\left[\Delta t \beta \Delta^{2} \bar{f}^{n}-S\left(\bar{f}^{n}\right)\right],
$$

where $\Delta t$ is the time step. The above discretized equation can be re-written as

$$
\bar{f}^{n+1}=\bar{f}^{n}+\left(I+\Delta t \beta \Delta^{2}\right)^{-1}\left[-\Delta t S\left(\bar{f}^{n}\right)\right] .
$$

The operator $\left(I+\Delta t \beta \Delta^{2}\right)^{-1}$ is smooth and able to suppress the unstable high wave number modes. The bi-Laplacian in Equation (3.9) is discretized using a second order accurate (in space) 13 point stencil centered-difference scheme. We found that $\beta=0.05$ results in a stable scheme for the time steps used in our calculations.

3.2. The IIM for the Stokes equations. Once the generalized surface tension $\sigma$ is calculated, we incorporate it into the boundary conditions for Stokes flow equations. We use an immersed interface method to solve the fluid field [41, 42]. The IIM was developed for solving interface problems with discontinuities across the interface. It is a second order accurate sharp interface method that takes into account the jump conditions directly into the difference stencil [41, 42].

In solving the fluid field, equations (2.24) and (2.25) are decomposed into three Poisson equations - one for the pressure and the other two for the velocity components $\mathbf{u}=\left(u_{x}, u_{y}\right)$. At time $t_{k}$, the equation for the pressure $p^{k}=p\left(\mathbf{x}, t_{k}\right)$ is obtained by taking the divergence of Equation (2.24). Because the viscosities of the fluids are matched, the pressure $p\left(\mathbf{x}, t_{k}\right)$ is a harmonic function,

$$
\nabla^{2} p^{k}=0
$$


with jump boundary conditions on the interface $\Sigma\left(t_{k}\right)$ :

$$
\left[p^{k}\right]_{\Sigma\left(t_{k}\right)}=-\left(\frac{1}{C a} \sigma \kappa\right)^{k}, \quad\left[\frac{\partial p}{\partial n}\right]_{\Sigma\left(t_{k}\right)}=\left(\frac{1}{C a} \nabla_{s}^{2} \sigma\right)^{k}
$$

and Neumann boundary conditions on $\partial \Omega$ :

$$
\left(\frac{\partial p}{\partial n}\right)^{k}=\left\{\begin{array}{l}
2\left(\nabla^{2} \mathbf{u} \cdot \mathbf{n}\right)^{k-1}-\left(\nabla^{2} \mathbf{u} \cdot \mathbf{n}\right)^{k-2}, k \geq 2, \\
\left(\nabla^{2} \mathbf{u} \cdot \mathbf{n}\right)^{k-1}, k=1 .
\end{array}\right.
$$

Once the pressure is determined, the velocity $\mathbf{u}^{k}$ is obtained by solving the Poisson system (recall $\lambda=1$ ):

$$
\nabla^{2} \mathbf{u}^{k}=\nabla p^{k}
$$

together with the jump boundary conditions on $\Sigma\left(t_{k}\right)[42]$ :

$$
\left[\mathbf{u}^{k}\right]_{\Sigma\left(t_{k}\right)}=0, \quad\left[\frac{\partial \mathbf{u}^{k}}{\partial n}\right]_{\Sigma\left(t_{k}\right)}=\left(\frac{1}{C a} \nabla_{s} \sigma\right)^{k},
$$

and the far-field Dirichlet boundary condition

$$
\mathbf{u}^{k}=\mathbf{u}_{\infty}^{k} \text { on } \partial \Omega .
$$

The Poisson equations for the pressure and velocity are discretized as follows. First, the grid points are divided into two groups. All grid points that are adjacent to the interface are classified as irregular grid points, while the remaining points are regular. At the regular grid points, a standard centered difference scheme is used to discretize the Poisson equations. At the irregular grid points, the standard center difference scheme is modified by adding a correction term to account for the jumps. The correction term, which modifies only the right hand side of the equation, can be derived from the two jump conditions, a Taylor series expansion of the solution in local coordinates, and the method of undetermined coefficients to yield a second order accurate discretization $[41,29]$. Note that, since $p^{k}$ is discontinuous across the interface, we can use the standard central finite difference scheme to approximate $\nabla p^{k}$ at the regular grid points. For irregular grid points, however, depending on the relative position of the points $\left(x_{i}, y_{i}\right),\left(x_{i+1}, y_{j}\right)$, and $\left(x_{i-1}, y_{j}\right)$, the approximation of $p_{x}^{k}$ (for example) is quite different from the regular grid point; see [29] for details.

The resulting scheme has local truncation error $O\left(h^{2}\right)$ at regular grid points (where $h$ is the spatial grid size), and $O(h)$ at irregular grid points. Since the whole set of irregular grid points is of co-dimension one, global second order accuracy can be achieved $[42,41]$. The resulting discrete systems are solved by using the FFT.

3.3. Interface capturing using the level-set function. The level-set method, first introduced by Osher and Sethian [43], has been highly successful in describing interface dynamics in many applications including multiphase flows; see the recent reviews $[44,45]$. Once the velocity field $\mathbf{u}^{k}$ is calculated from solving the Stokes equations, the level-set method is implemented to capture the interface position. Since the interface moves with the fluid, we take

$$
\frac{\partial \phi}{\partial t}+\mathbf{u} \cdot \nabla \phi=0
$$


The interface $\Sigma\left(t_{k}\right)$ is represented by the zero set of the level-set function $\phi^{k}=\phi\left(\mathbf{x}, t_{k}\right)$, i.e. $\Sigma\left(t_{k}\right)=\left\{\mathbf{x}: \phi\left(\mathbf{x}, t_{k}\right)=0\right\}$. Assume that $\{\mathbf{x}: \phi(\mathbf{x}, t)<0\}=\Omega_{1}$, so that the outward normal and curvature are

$$
\mathbf{n}^{k}=\frac{\nabla \phi^{k}}{\left|\nabla \phi^{k}\right|}, \quad \kappa^{k}=\nabla \cdot\left(\frac{\nabla \phi^{k}}{\left|\nabla \phi^{k}\right|}\right)
$$

The level-set function is re-initialized after each time step to be a signed distance function locally near the interface [46]. This is performed by solving the following Hamilton-Jacobian equation to steady state:

$$
\left\{\begin{array}{l}
\phi_{\tau}+S\left(\phi_{0}\right)(|\nabla \phi|-1)=0, \\
\phi(\mathbf{x}, 0)=\phi_{0}(\mathbf{x}),
\end{array}\right.
$$

where $\phi_{0}$ is the level set function before the re-initialization, $\tau$ is the pseudo-time, and $S(x)$ is the sign function of $x$ defined as

$$
S(x)=\left\{\begin{array}{lll}
-1, & \text { if } & x<0 \\
0, & \text { if } & x=0 \\
1, & \text { if } & x>0 .
\end{array}\right.
$$

In practice, the re-initialization is performed at every time step.

In the above equations, the standard third order upwind WENO method [47] is used for the spatial discretization and the standard third order TVD Runge-Kutta method [48] is used for time stepping. A smoothed approximation of the sign function (3.20) is used,

$$
\tilde{S}(\phi)=\frac{\phi}{\sqrt{\phi^{2}+h^{2}}},
$$

where as before $h$ is the spatial grid size.

3.4. Enforcing area and surface mass conservation. One of the drawbacks of the level-set method is that area of the enclosed vesicle is not exactly conserved by the flow. In addition, the mass of $f$ is not exactly conserved by our algorithm either. Typically, small errors are incurred at every time step, and after long times these errors may accumulate and lead to inaccurate results. Interestingly, we find that the most significant source of mass error arises from the fact that the the discrete velocity field obtained from the IIM is not essentially divergence free [29]. Therefore, to enforce area conservation, a small correction is added to the normal velocity of moving interface at every time step. This is an approach frequently used in boundary integral simulations (e.g. see [49]). Let $\tilde{\mathbf{u}}_{h}$ be the discrete velocity obtained from the IIM. We determine a small correction $\alpha$ to ensure that the net mass flux across the interface is zero:

$$
\int_{\Sigma}\left(\tilde{\mathbf{u}}_{h}+\alpha \mathbf{n}\right) \cdot \mathbf{n} d s=0
$$

This yields the explicit expression

$$
\alpha=-\frac{\int_{\Sigma} \tilde{\mathbf{u}}_{h} \cdot \mathbf{n} d s}{\int_{\Sigma} d s}=-\frac{\int \tilde{\mathbf{u}}_{h} \cdot \mathbf{n} \delta_{\Sigma}(\phi) d \mathbf{x}}{\int \delta_{\Sigma}(\phi) d \mathbf{x}} .
$$


The modified velocity is then used to advect the level set function and the surface mass concentration $f$. The modification above ensures that the total mass flux across the interface is zero and is found to result in dramatically reduced mass loss overall [29].

The above modified velocity also improves the conservation of $f$, however there is still significant loss of total surface mass over long-times due to numerical diffusion. The simplest way to enforce this correction is to multiply the mass concentration $f$ by a constant factor to ensure that total surface mass is conserved. Let $\tilde{f}_{h}$ be the solution of the discrete surface mass concentration equation and let $f_{0}, \phi_{0}$, and $\Sigma_{0}$ be the initial mass concentration, level-set function, and interface respectively. Then, we choose $\beta$ such that

$$
\int_{\Sigma} \beta \tilde{f}_{h} d \Sigma=\int_{\Sigma_{0}} f_{0} d \Sigma_{0}
$$

which yields

$$
\beta=\frac{\int_{\Sigma_{0}} f_{0} d \Sigma_{0}}{\int_{\Sigma} \tilde{f}_{h} d \Sigma}=\frac{\int_{\Omega} f_{0} \delta_{\Sigma_{0}} d \mathbf{x}}{\int_{\Omega} \tilde{f}_{h} \delta_{\Sigma} d \mathbf{x}} .
$$

The mass concentration is then reset to be $f_{h}=\beta \tilde{f}_{h}$. We refer the reader to [38] for numerical approximations of the delta function in the above integrals, noting that $\delta_{\Sigma}=\delta(\phi)|\nabla \phi|$.

Finally, we note that other, more sophisticated area and surface mass concentration corrections can be derived that take into account the interface curvature and mass concentration gradients, etc. Nevertheless, we found it sufficient to use the simpler corrections described above.

\section{Numerical results}

In this section, we present 2D simulations illustrating the effect of flow, surface tension, and phase decomposition on the dynamics of a vesicle in viscous fluids. We focus our study on physical aspects of the problem. The detailed convergence studies of a similar algorithm for a viscous drop with surfactant are presented in [29]. We first study the case without the arclength constraint, i.e. when the interface is allowed to relax freely. We then consider the effects of the arclength constraint in the following sections. Note that in these calculations, we do not apply an external flow. The flow field is generated by the deformation and motion of the vesicle.

Unless otherwise noted, the computational domain is $\Omega=[-1,1] \times[-1,1]$. A uniform Cartesian grid is used with $h_{x}=h_{y}=h=0.01$ for all simulations. The time step is set to be $\Delta t=h / 10$. The Peclet number is $P e=1$. We take the chemical free energy to be $g(\bar{f}, f)=\left(\frac{f}{\bar{f}}\right)^{2}\left(1-\frac{f}{\bar{f}}\right)^{2} / 4$ which is a modified double-well potential. That is, the states $f=0$ and $f=\bar{f}$ are energetically preferred by the chemical energy. We take the surface energy density in Equation (2.12) to be $\gamma(f, \bar{f})=\exp (-x f / \bar{f})$, where $x$ measures the reduction in surface tension by the phase $f=\bar{f}$. Here we let $x=0.2$, so the surface tension $\tau$ of the two phases are not matched, and $\tau(0)=1>\tau(\bar{f})=\exp (-0.2)=0.8187$ by Equation (2.22). We take the Capillary number $C a=1.0$, the Cahn number $\mathcal{C}=0.02$, and the Mach number $\mathcal{M}=1$. The parameter $\bar{a}$ used in Equation (3.5) is set to be $\bar{a}=0.25$. The initial concentration field on the interface is a small perturbation of the unstable state 0.5 . In particular, we take $f(x, y, 0)=0.5+0.01(\sin x \cos y+\sin (4 x) \cos (3 y))$, so the initial phase distribution is symmetric about the $x$-axis. At $t=0, \bar{f}(s, 0)=1$. 
4.1. Relaxation dynamics of an ellipse without arclength constraint.

The initial vesicle shape is taken to be an ellipse located at the origin, $x^{2}+2 y^{2}=$ 0.25 . Without the arclength constraint, it is expected that the interface will relax freely back to a circle (with minimum surface energy). Note that the initial ellipse has a small aspect ratio, i.e. the ratio between the long axis and the short axis is $a / b=\sqrt{2}$. The arclength at $t=0$ is $L_{\text {ellipse }} \approx 2.7$. The arclength for a circle with the same area is $L_{\text {ellipse }} \approx 2.642$. So there is only a small amount of excess arclength, $\Delta L=L_{\text {ellipse }}-L_{\text {circle }} \approx 0.058$.

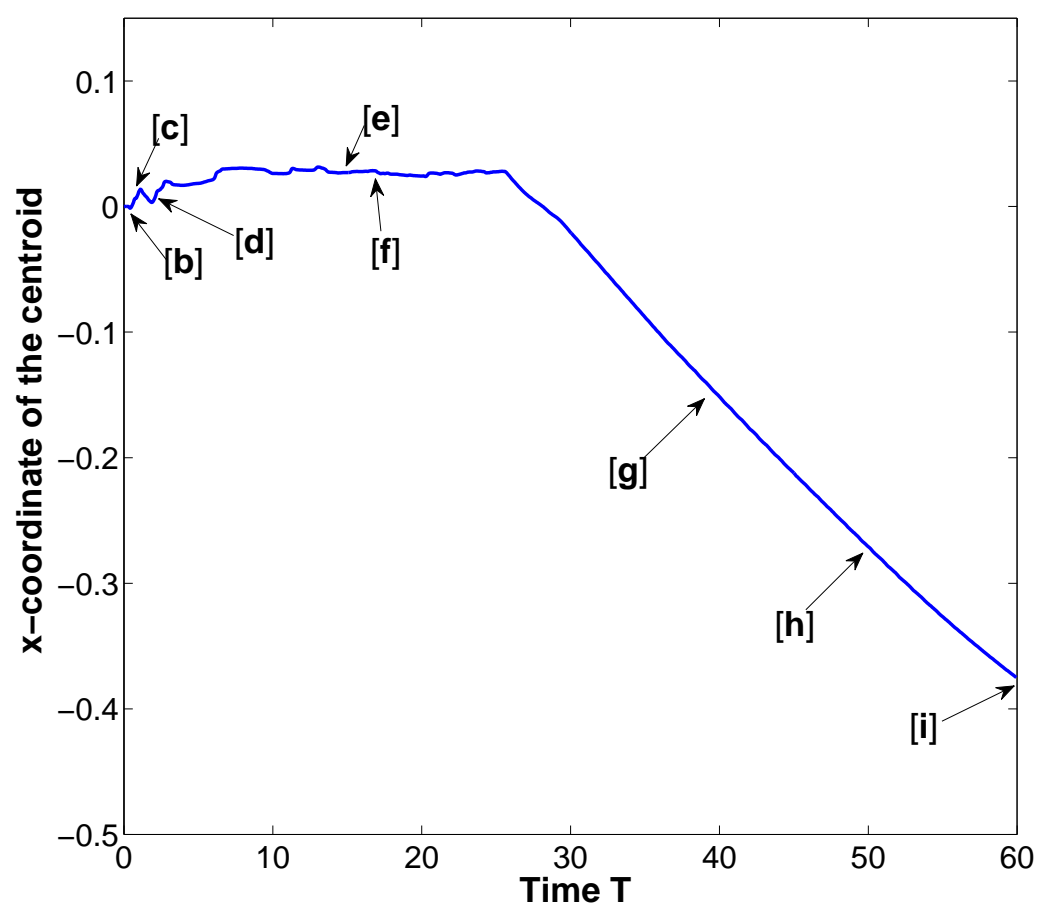

FIG. 4.1. The $x$-coordinate of the centroid of the moving vesicle. The initial shape is an ellipse with $x^{2}+2 y^{2}=0.5$. Distribution of mass concentration $f$ along the interface, morphologies of the vesicle, and the streamlines of the velocity field for $x=0.2, C a=1.0, P e=1.0, \mathcal{C}=0.02$, and $\mathcal{M}=1.0$ are marked at different times.

In Figure (4.1)[a], we plot the $x$-coordinate of the vesicle centroid and an evolution sequence indicated by capital letters at the indicated times. At early times, the vesicle oscillates around the origin. At later times, the vesicle moves to the left at a constant velocity, see the straight line after $t=27$, until late times when the vesicle interacts with the wall.

In Figure(4.1)[b]-[i], we show the detailed distribution of the mass concentration $f$ along the interface, morphologies of the vesicle, and streamlines of the velocity field during the dynamics. We plot the concentration $f$ along the interface using different colors. The color scale ranges from $f=0$ (blue) to $f=\bar{f}$ (red). We set the background color to be 0.3 in order to provide a contrast. At $t=0$, the interface at the high curvature tip region retracts rapidly to the center while the low curvature 


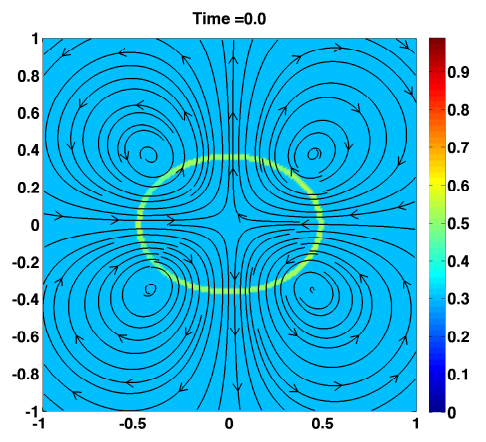

[b]
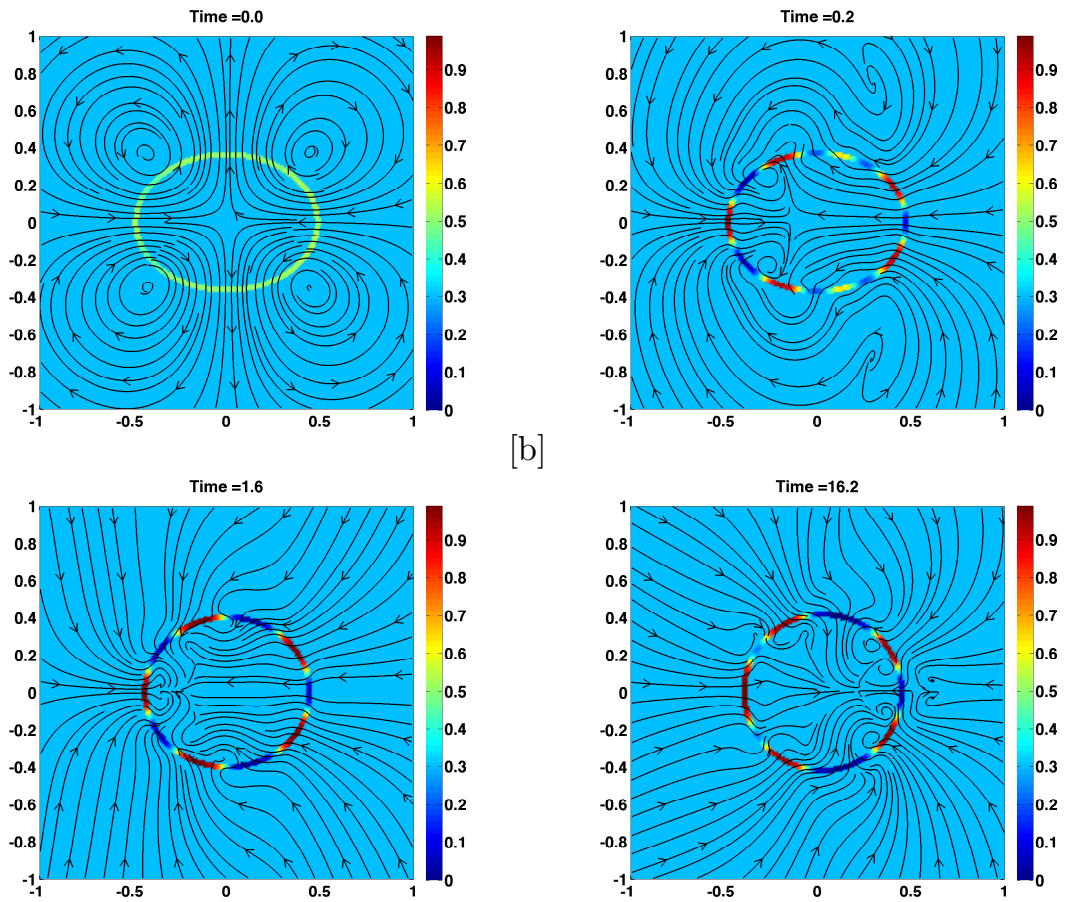

[d]

[c]
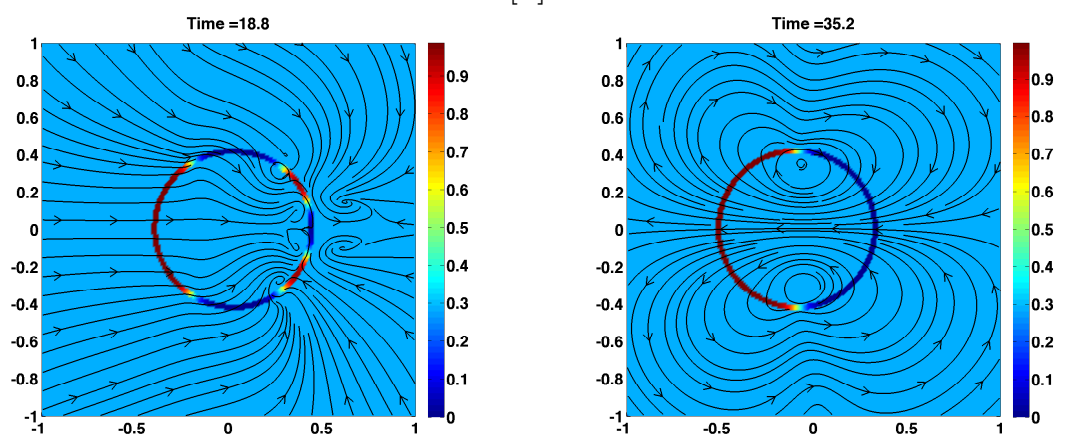

$[\mathrm{f}]$

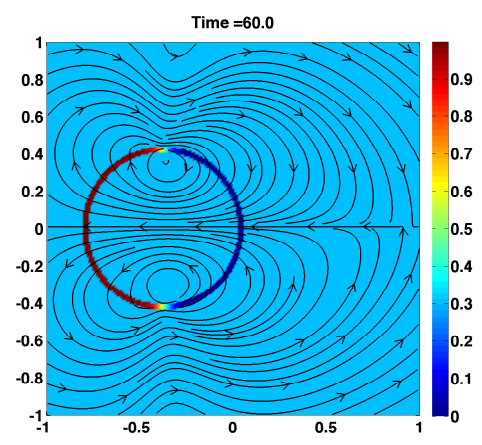

$[\mathrm{g}]$

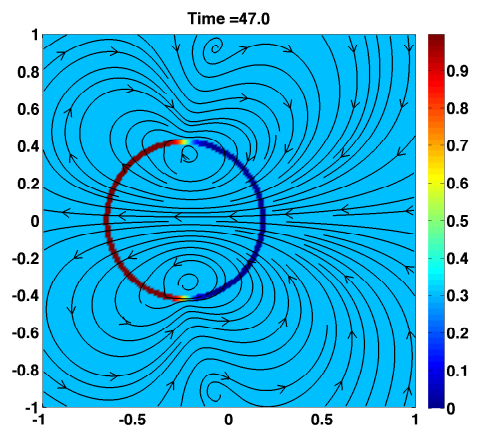

$[\mathrm{h}]$

$[\mathrm{e}]$

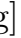

FIG. 4.1. Distribution of mass concentration $f$ along the interface, morphologies of the vesicle, and the streamlines of the velocity field at times $t=0$ [b], 0.2 [c], $1.6[d], 16.2[e], 18.8[\mathrm{f}], 35.2[\mathrm{~g}]$, $47.0[\mathrm{~h}], 60.0[\mathrm{I}]$. 


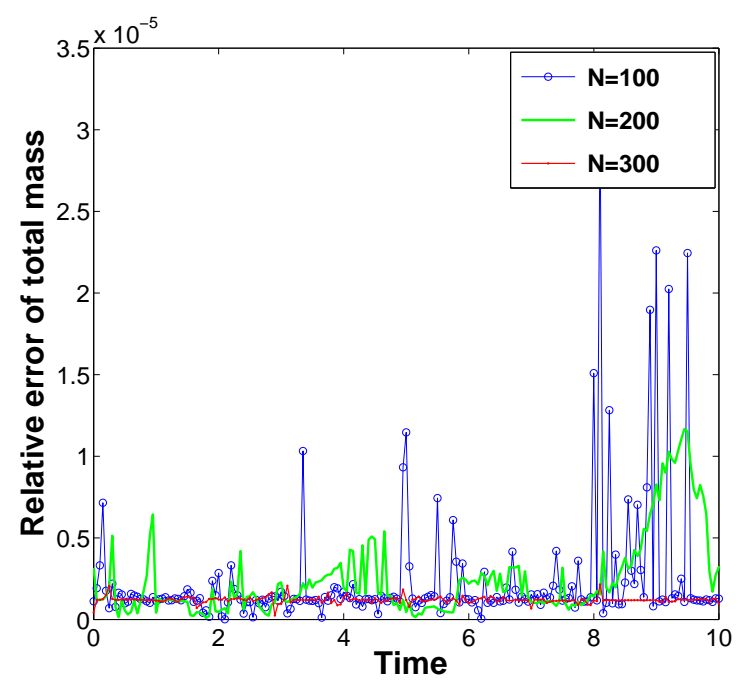

FIG. 4.2. Convergence of the surface phase concentration, the total mass $\bar{f}$.

region expands from the center. Similar to a Taylor four-roll mill [50], the relaxation dynamics produces four vortex-like rotational flows around the tips shown in Figure $4.1[\mathrm{~b}]$, in which the streamlines point inward to the center along the horizontal axis $(y=0)$ and point outward along the vertical axis $x=0$. Here $x$ and $y$ refer to global Cartesian coordinates axis centered at the origin.

The relaxation process continues for a short time until the surface tension brings the ellipse back to a circular shape; see Figure $4.1[\mathrm{~d}]$ at $t=1.6$. It is evident that the mass mixture decomposes at $t=0.2$ in Figure $4.1[\mathrm{c}]$. At $t=1.6$, the first round phase decomposition is finished and there are regions with well-separated phases $f=0$ (low concentration) and $f=\bar{f}$ (high concentration). Note that due to the nonlinear coupling between the shape and flow, the phase energy is reduced further by a second round phase decomposition (from Figure 4.1[e] at $t=16.2$ to Figure $4.1[\mathrm{~g}]$ at $t=26.8$ ). This process eliminates extra eight phase boundaries (i.e. the places where $f=0$ phase(blue) and $f=\bar{f}$ phase(red) are met). Clearly, after the second round phase decomposition, the vesicle has only two regions with phase $f=0$ on the right half and $f=\bar{f}$ on the left half.

The phase decomposition is accompanied by horizontal translation dynamics, i.e. the vesicle moves to the left. For example, in Figure 4.1[g] at $t=35.2$ the directions of the streamlines are pointing to the left along $x$-axis. This is because the surface tension of phase $f=0$ is larger than that of the $f=\bar{f}$ phase on the left, i.e. $\tau(0)=$ $1>\tau(\bar{f})=\exp (-0.2)=0.8187$. So the forces along the $x$-axis are not balanced and the total net force (with a direction pointing to the left) drives the vesicle to move toward the left. This is clearly seen in Figure 4.1[h] and Figure (4.1)[i], in which the centroid of the vesicle keep moving to the left along the negative $x$-axis indicating the horizontal translation of the vesicle. Interestingly, at later times the vesicle travels at a constant velocity; see the straight line after $t=27$. This behavior resembles the case that a particle is falling in the viscous fluid by its own weight, in which a constant velocity is reached at later time [51]. Around the phase boundaries where phase $f=0$ 
and phase $\bar{f}$ meet, rotational flow fields are produced both inside and outside the vesicle. These vortexes are traveling with the vesicle. Recall that we do not apply any external flow in this simulation. The locomotion of the membrane is completely due to the coupling between the flow and imbalance of surface tension forces.

Next, we examine the numerical errors associated with these calculations. Without the arclength constraint, the arclength shrinks quickly at early times and settles down at a value which is approximately $2.2 \%$ smaller than the initial value, which is the excess amount of arclength considered in the initial ellipse. The relative error in mass of surface phase $f$ and total mass $\bar{f}$ are measured using $\left|\int_{\Sigma(t)} f(s, t) d \Sigma-M_{f}(0)\right| / M_{f}(0)$ and $\left|\int_{\Sigma(t)} \bar{f}(s, t) d \Sigma-M_{t o t}(0)\right| / M_{t o t}(0)$ respectively. The maximum value of the relative errors are very small, approximately $0.025 \%$ for $f$ and $0.004 \%$ for $\bar{f}$, indicating that the material field is well conserved. In Figure (4.2), we show a sample convergence study in space for the total mass conservation during evolution. Similar convergence (not shown) is observed for computations shown in Figure (4.3) and (4.4).

\subsection{Relaxation dynamics of an ellipse with arclength constraint.}

We next investigate the effect of the arclength constraint. We use the same initial conditions as those used to compute Figure 4.1 and set the parameter $\lambda=100$ to enforce the global arclength constraint.

In Figure 4.3[a], we plot the $x$-coordinate of the vesicle centroid and present an evolution sequence describing the morphology, phase distribution, and the velocity field by streamlines in Figure 4.3[b]-[k]. Similar to the unconstrained case, the vesicle moves to the left because the surface tension force is not balanced and there is a net force pointing to the left. At $t=0.0$ the vesicle retracts to produce a Taylor four-roll mill structure. The surface mass mixture starts to decompose quickly and the first round phase decomposition finishes around $t=1.6$; see Figure $4.3[\mathrm{~b}]-[\mathrm{d}]$. The morphology, however, stays roughly elliptical, which is different from the unconstrained case shown in Figure 4.1[d], where the shape relaxes back to a circle at $t=1.6$. A second round of phase decomposition starts at $t=12$, as shown Figure 4.3[e], and finishes around $t=16.2$ after eliminating four phase boundaries, shown in Figure 4.3[f], in which most of the low concentration phase $f=0$ is in the right part of the vesicle, although a small amount remains trapped in the left region. This is very different from the unconstrained case where all of the low concentration $f=0$ phase is accumulated at the right half of the circular vesicle.

Wrinkles on the vesicle boundary start to develop in the high concentration phases; see the red regions in Figure 4.3[f]. The wrinkles become evident in Figure $4.3[\mathrm{~g}]-[\mathrm{k}]$. This is due to the arclength constraint, and the vesicle is not allowed to fully relax back to a circular shape. The excess arclength is manifested as wrinkles in the high concentration phases, where the surface tension is smaller than that of the low concentration regions.

Membrane wrinkling has been previously observed in experiments of elastic capsules in an applied shear flow by Walter et al. [17]. Finken and Seifert [18] performed a mathematical analysis and derived analytical results both for the threshold value of the shear rate and for the critical wavelength of the wrinkling. Wrinkling has also been observed in experiments of vesicles by Kantsler et al. [19] under time-dependent elongation flow [19]. It has been suggested that the winkling phenomena are related to the dynamical instability induced by negative surface tension of the membrane [20] during the vesicle deflation. These wrinkles usually are associated with higher 


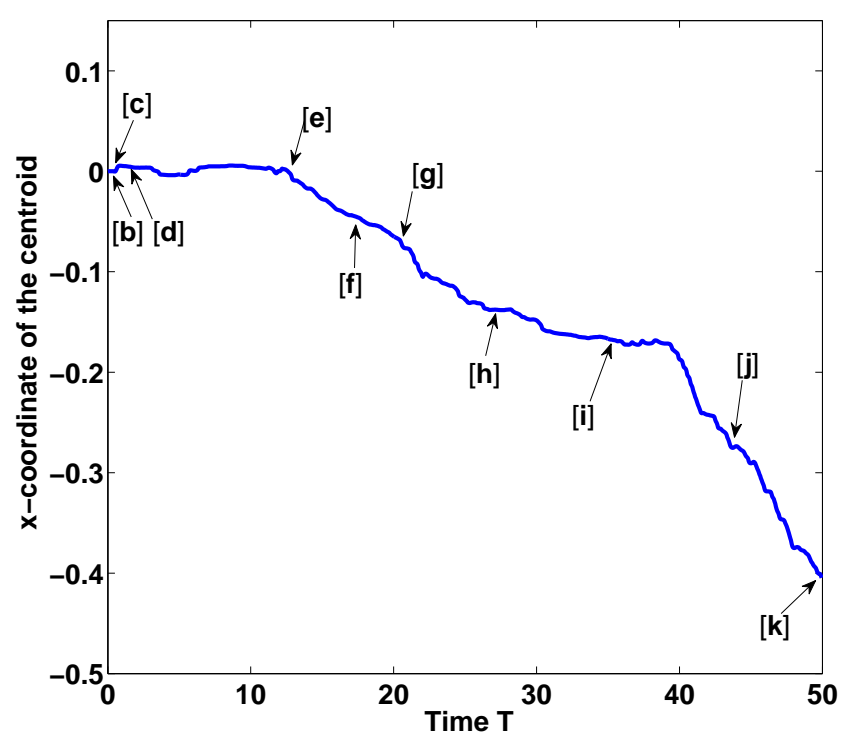

FIG. 4.3. The $x$-coordinate of the centroid of the moving vesicle. The initial shape is an ellipse with $x^{2}+2 y^{2}=0.5$. Distribution of mass concentration $f$ along the interface, morphologies of the vesicle, and the streamlines of the velocity field for $x=0.2, C a=1.0, P e=1.0, \mathcal{C}=0.02$, and $\mathcal{M}=1.0$ are marked at different times.

order modes. After a careful check of our surface tension, we indeed found that the development of the wrinkling instability in the high concentration regions is closely associated with negative surface tension. As an example, in Figure 4.3[1], we plot the surface tension versus the arclength at time $t=16.2$, when the wrinkles are about to develop. Corresponding morphology and phase field are shown in Figure 4.3[f]. It is evident that the surface tension is negative at high concentration regions and there are rapid and smooth transitions across the phase boundaries. A detailed study using a more accurate boundary integral method is in preparation [52].

During the evolution, the maximum relative error of vesicle arclength is very small $(0.041 \%)$ when compared with the unconstrained case $(2.2 \%)$, indicating that the arclength is conserved well. We have tested other $\lambda$ 's and found that large values of $\lambda$ result in a better conservation of the arclength. Similar to the previous studies, the relative error in mass of surface phase $f$ and total mass $\bar{f}$ are both very small, approximately $0.025 \%$ for $f$ and $0.002 \%$ for $\bar{f}$ indicating, the material field is also well conserved.

4.3. Relaxation dynamics of an elongated ellipse with arclength constraint. In this section, we investigate the evolution of an elongated ellipse, $x^{2}+8 y^{2}=0.25$. Note that the ellipse has a larger aspect ratio, $a / b=2 \sqrt{2}$. The arclength at $t=0$ is about $L_{\text {ellipse }} \approx 2.115$. The arclength for a circle with the same area is $L_{\text {ellipse }} \approx 1.868$, so there is a large amount of excess arclength, $\Delta L=L_{\text {ellipse }}-L_{\text {circle }} \approx 0.247$. We set $\lambda=100$ and all other parameters are the same as those used to produce Figure 4.3.

In Figure 4.4[a], we plot the $x$-coordinate of the vesicle centroid and show an 

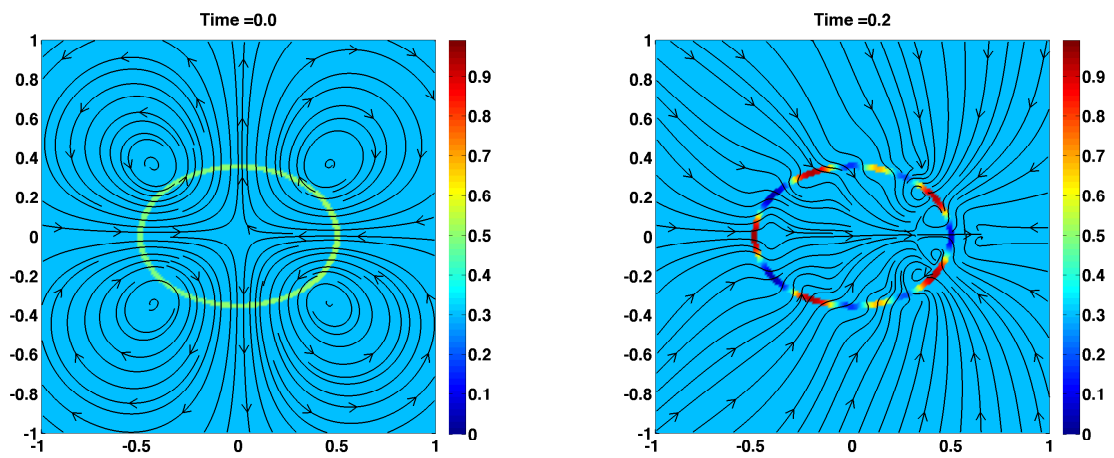

[b]
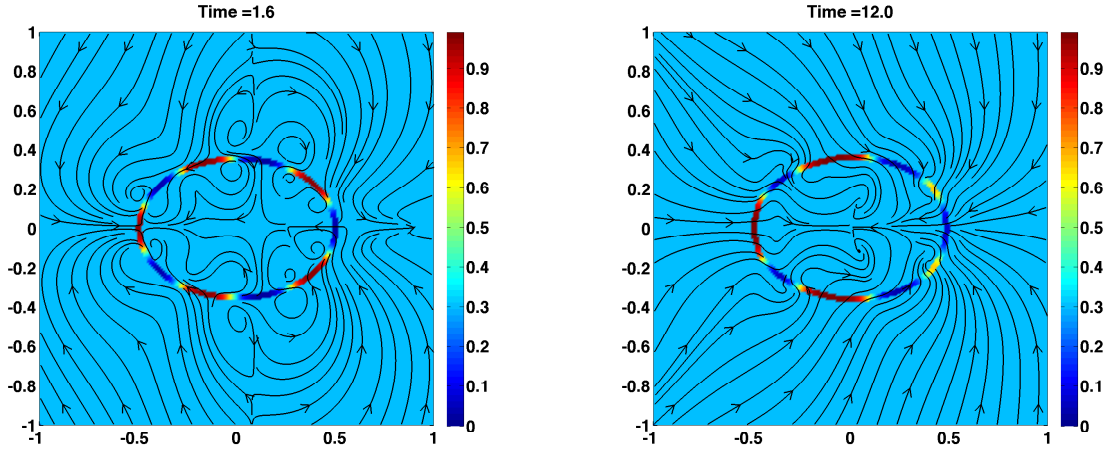

[d]
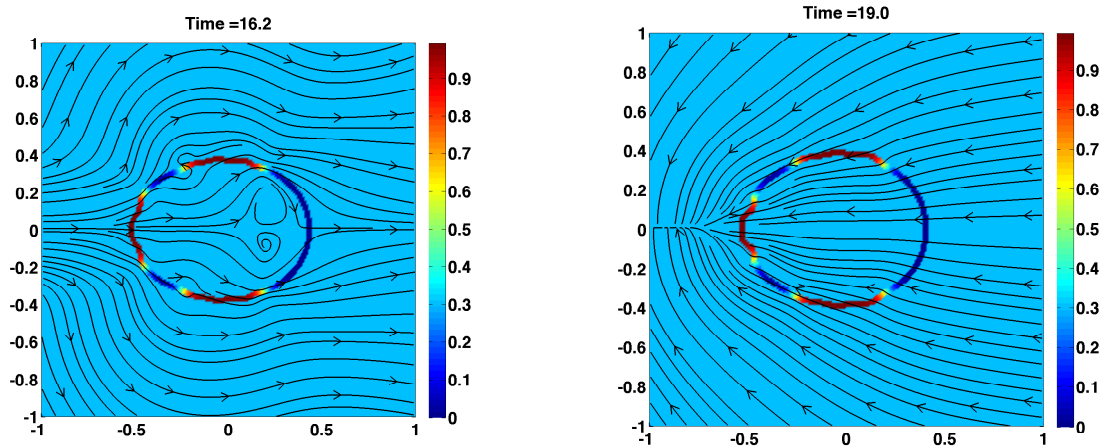

$[\mathrm{f}]$

FIG. 4.3. Distribution of mass concentration $f$ along the interface, morphologies of the vesicle, and the streamlines of the velocity field at times $t=0[b], 0.2[c], 1.6[d], 12.0[e], 16.2[f]$, and 19.0 $[g]$.

evolution sequence with time indicated in the caption. Unlike the previous two cases, the vesicle mainly oscillates around the origin. There are also two rounds of phase decompositions during early times; see Figure 4.4[b]-[g]. Note that after the phase decomposition, the high concentration phase is localized in the high curvature regions and the low concentration phase emerges in the low curvature regions. There are four phase boundaries along the interface. The streamlines of velocity field during early times all point inward to the center, indicating retraction of the vesicle. However, 

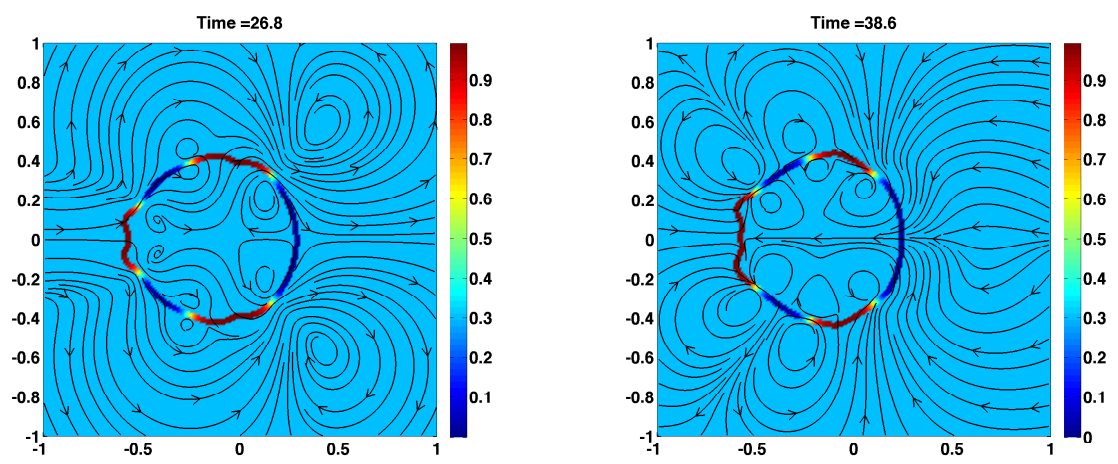

$[\mathrm{h}]$
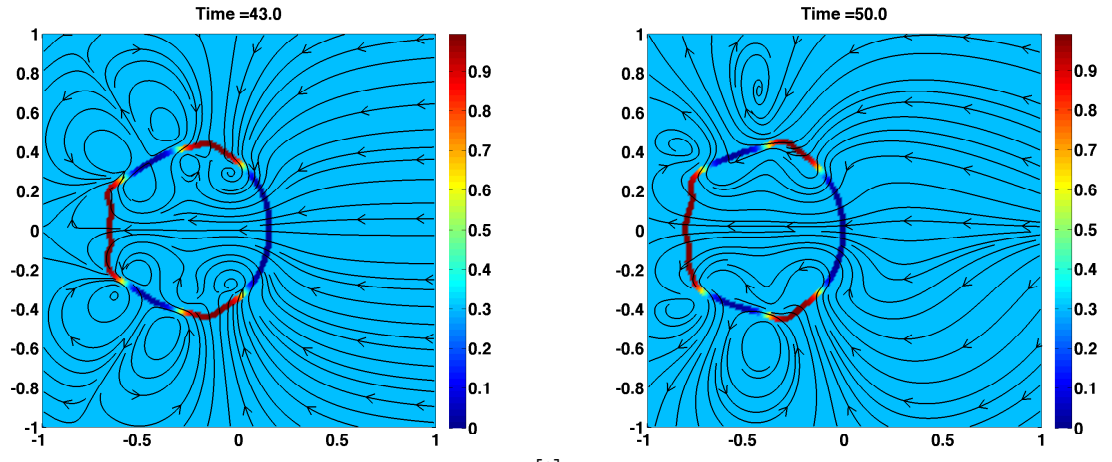

[j]

$[\mathrm{k}]$

FiG. 4.3. Distribution of mass concentration $f$ along the interface, morphologies of the vesicle, and the streamlines of the velocity field at times $t=26.8[\mathrm{~h}], 38.6[\mathrm{i}], 43.0[\mathrm{j}]$, and $50.0[\mathrm{k}]$.

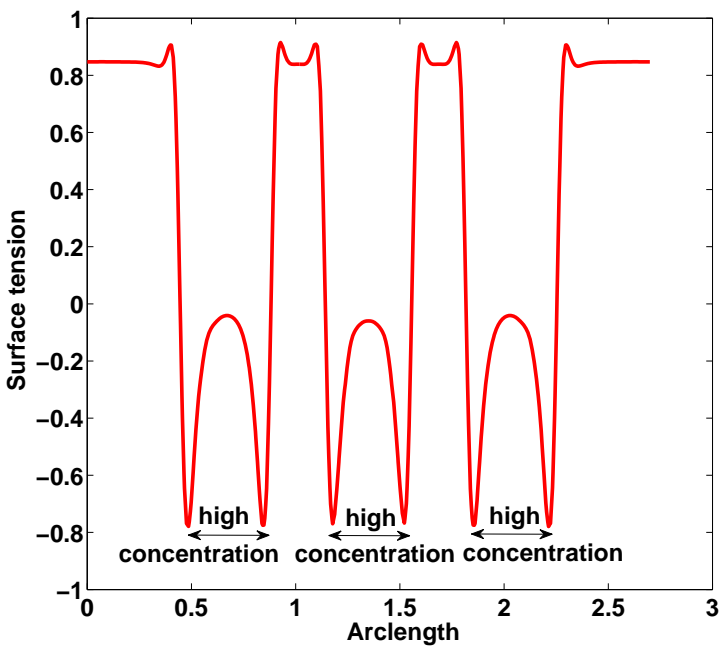

FIG. 4.3. Negative surface tension in high concentration regions [l]. 
the vesicle remains in a roughly elliptical shape. It seems the arclength constraint successfully limits the free-relaxation of the vesicle. At later times, the vesicle shape continues to evolve and forms two small buds around the tip regions; see Figure 4.4[h][i]. The bud on the right half of the vesicle expands and grows larger at the expense of the left one. At $t=14$, a neck connecting the bud and the right half of the interface forms. Note that there are also wrinkles observed during this process (see Figure $4.4[\mathrm{j}])$, but they are not stationary and are only excited for a short amount of time.

The bud formation is also accompanied by a horizontal translation because of the imbalance of the surface tension forces. There are also rotational flows produced at the phase boundaries during the evolution. With arclength constraint $\lambda=100$, the arclength is well preserved with only a $0.04 \%$ loss compared with the initial value.

Bud formation has been studied in multicomponent vesicle dynamics with bending energy included; e.g. see $[11,28,53,16]$. It has also been reported recently that a time-dependent flow with a switch in the direction of the velocity gradient can also lead to bud formation [6]. Here our flow field is far more complicated than the elongation flow. Further, in our simulation, there is no bending force involved and it is the nonlinear interactions among the flow, the surface phases, and the variable surface tension forces that give rise to the bud formation.

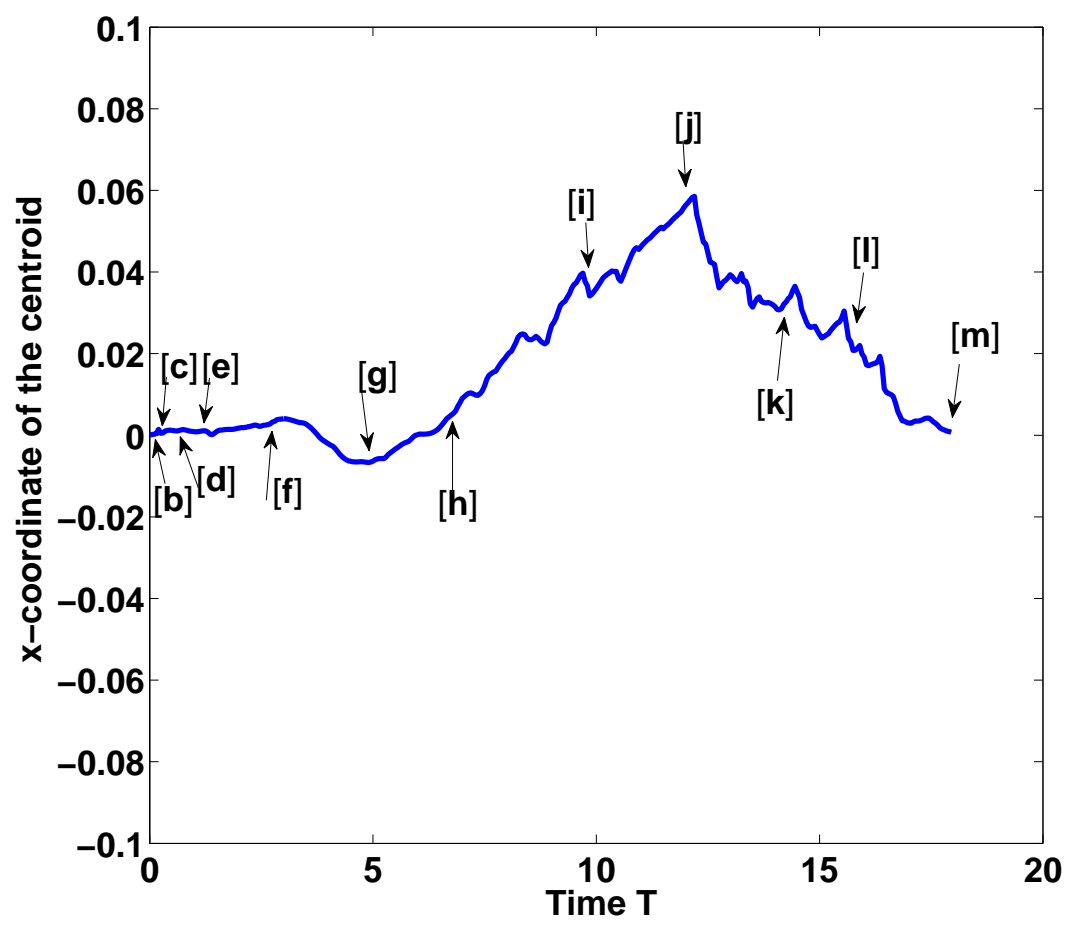

FIG. 4.4. The $x$-coordinate of the centroid of the moving vesicle. The initial shape is an ellipse with $x^{2}+8 y^{2}=0.5$. Distribution of mass concentration $f$ along the interface, morphologies of the vesicle, and the streamlines of the velocity field for $x=0.2, C a=1.0, P e=1.0, \mathcal{C}=0.02$, and $\mathcal{M}=1.0$ are marked at times different times. 

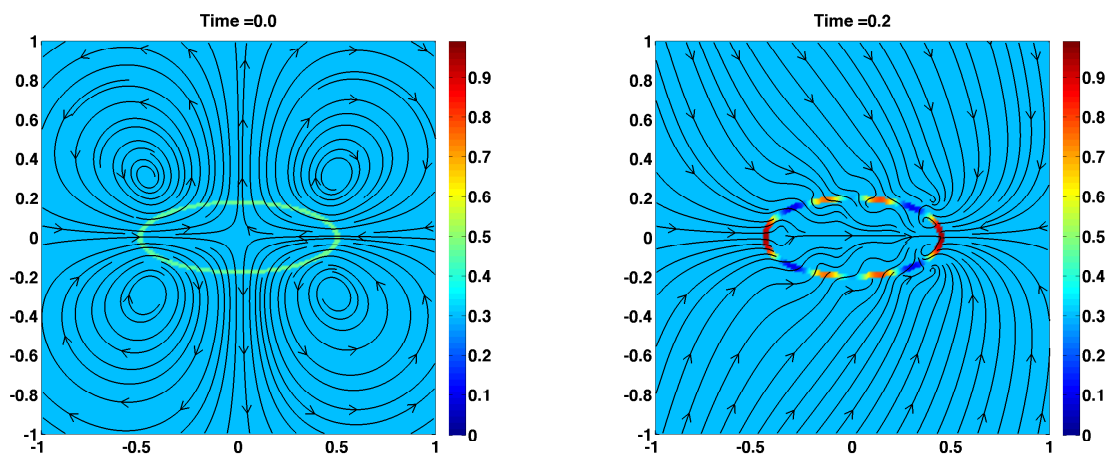

[b]
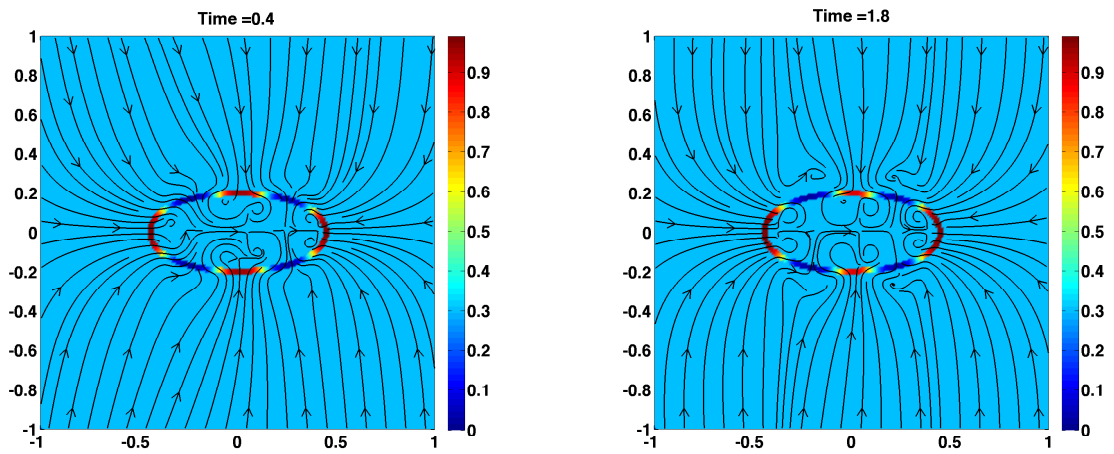

[d]
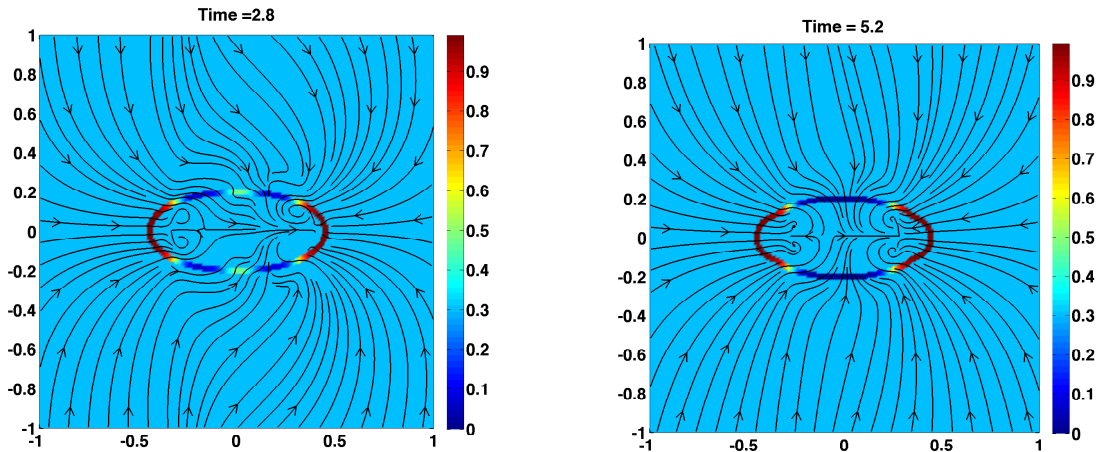

$[\mathrm{f}]$

$[\mathrm{g}]$

FIG. 4.4. Distribution of mass concentration $f$ along the interface, morphologies of the vesicle, and the streamlines of the velocity field at times $t=0[b], 0.2[c], 0.4[d], 1.8[e], 2.8[\mathrm{f}]$, and $5.2[\mathrm{~g}]$.

\section{Conclusions and future work}

We have developed a thermodynamically consistent model of a multicomponent membrane using a simplified version of the Helfrich model. To investigate the effects of surface tension, we neglect the bending forces and spontaneous curvature. Using an energy variation approach, we derived constitutive equations for: (1) the generalized surface tension forces imparted to the flow as a boundary condition at the membrane surface; (2) the diffusion flux imparted in the mass conservation equations of CahnHilliard type on the evolving membrane surface. This simplified model is capable 

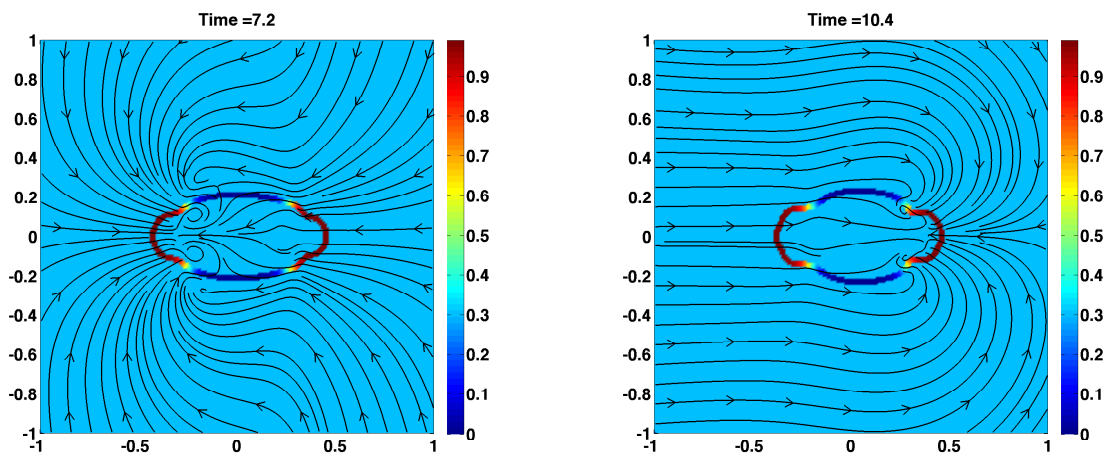

$[\mathrm{h}]$

[i]
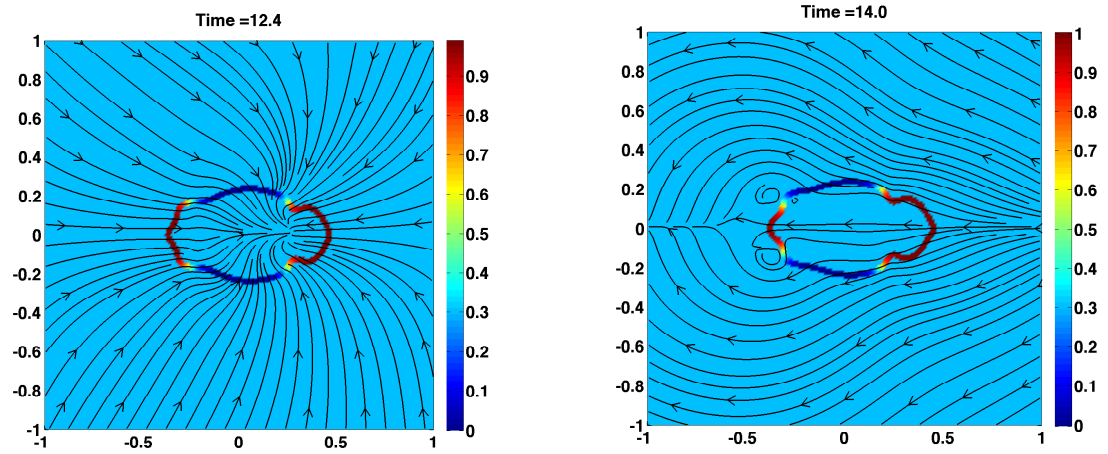

[j]
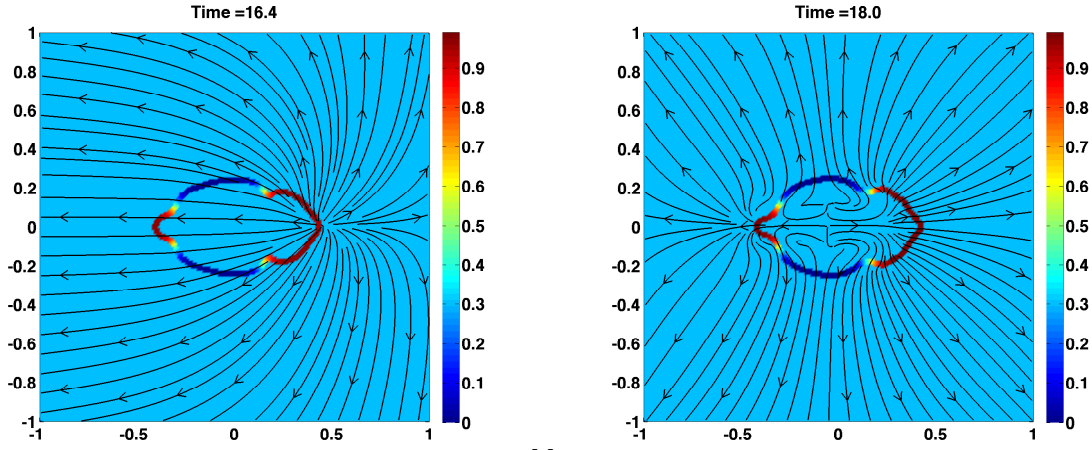

FIG. 4.4. Distribution of mass concentration $f$ along the interface, morphologies of the vesicle, and the streamlines of the velocity field at times $t=7.2[h], 10.4[i], 12.4[j], 14.0[k], 16.4[l]$, and $18.0[\mathrm{~m}]$.

of describing the nonlinear coupling among the flow, vesicle morphology, and the evolution of the surface phases.

To solve the highly nonlinear coupled system, a new numerical method is presented along the lines of the methods developed for interfacial flows with surfactant [29]. This method combines the immersed interface method to solve the flow equations, and the Laplace-Young jump conditions, with the level-set method to represent and evolve the interface and a non-stiff Eulerian algorithm to update the mass concentration on the interface. Our numerical results on the dynamics of a two-dimensional 
vesicle reveal that nonhomogeneous surface tension, coupled with the surrounding viscous fluid, introduces vesicle locomotion and budding. When the arclength constraint is not present, an initially perturbed circular vesicle evolves back to a circular shape with well-separated phases. Because of the imbalanced surface tension between two phases, vesicles move in the direction that the surface tension is small. When the arclength constraint is enforced, in addition to self-locomotion, a perturbed circular vesicle exhibits various morphologies during its evolution. Examples include the formation of wrinkles for vesicles with limited excess arclength and buds for vesicles with larger excess arclength.

In the future, we plan to implement a boundary integral method to further investigate and quantify the effects of surface tension using Lagrange multipliers to enforce the local surface area constraint. We also plan to include bending forces and spontaneous curvature to make the model more realistic. We note that, because of the higher nonlinear terms $\kappa_{s s}$ introduced by the bending energy, an adaptive grid method has to be used to compute this term accurately, especially around the regions with high curvatures. Theoretical studies of variable surface tension are also being performed.

Acknowledgments. The authors thank Drs. Jinsun Sohn and Jianjun Xu for useful discussions. SL acknowledges support from the National Science Foundation, Division of Mathematical Sciences (NSF-DMS) grant DMS-0914923. JL also acknowledges partial support from NSF-DMS.

\section{REFERENCES}

[1] R. Lipowsky and E. Sackman, Structure and Dynamics of Membranes-From Cells to Vesicles, Elsevier, Amsterdam, 1A/B, 1995.

[2] R. Lipowsky, The conformation of membranes, Nature, 349-475, 1991.

[3] B. Alberts, D. Bray, J. Lewis, M. Raff, K. Roberts, and J.D. Watson, Molecular biology of the cell, Garland, New York, 1994.

[4] H.T. McMahon and J.L. Gallop, Membrane curvature and mechanisms of dynamic cell remodelling, Nature, 438, 590-596, 2005.

[5] T. Baumgart, S.T. Hess, and W.W. Webb, Imaging coexisting fluid domains in biomembrane models coupling curvature and line tension, Nature, 425, 821-824, 2003.

[6] J. Deschamps, V. Kantsler, E. Segre, and V. Steinberg, Dynamics of a vesicle in general flow, PNAS, 106(28), 11444-11447, 2009.

[7] F. Julicher and R. Lipowsky, Shape transformations of vesicles with intramembrane domains, Phys. Rev. E, 53, 2670-2683, 1996.

[8] D.R. Nelson, T. Piran, and S. Weinberg, Statistical Mechanics of Membranes and Surfaces, World Scientific, Singapore, 2004.

[9] C. Pozrikidis, Interfacial dynamics for Stokes flow, J. Comp. Phys., 169(2), 20, 250-301, 2001.

[10] C. Pozrikidis, Boundary Integral and Singularity Methods for Linearized Viscous Flow, Cambridge University Press, Cambridge, 1992.

[11] T. Taniguchi, Shape deformation and phase separation dynamics of two-component vesicles, Phys. Rev. Lett., 76(23), 4444-4447, 1996.

[12] Q. Du, C. Liu, and X. Wang, Simulating the deformation of vesicle membranes under elastic bending energy in three dimensions, J. Comp. Phys., 212(2), 1, 757-777, 2006.

[13] S.K. Veerapaneni, D. Gueyffier, D. Zorin, and G. Biros, A boundary integral method for simulating the dynamics of inextensible vesicles suspended in a viscous fluid in 2d, J. Comp. Phys., 228(7), 2334-2353, 2009.

[14] S.K. Veerapaneni, D. Gueyffier, G. Biros, and D. Zorin, A numerical method for simulating the dynamics of $3 d$ axisymmetric vesicles suspended in viscous flow, J. Comp. Phys., 228(19), 20, 7233-7249, 2009.

[15] A. Rahimian, S.K. Veerapaneni, and G. Biros, Dynamic simulation of locally inextensible vesicles suspended in an arbitrary two-dimensional domain, a boundary integral method, J. Comp. Phys., 229(18), 1, 6466-6484, 2010. 
[16] J. Sohn, Y. Tseng, S. Li, J. Lowengrub, and A. Voigt, Dynamics of multicomponent vesicles in a viscous fluid, J. Comp. Phys., 229(1), 1, 119-144, 2010.

[17] A. Walter, H. Rehage, and H. Leonhard, Shear induced deformation of microcapsules: Shape oscillations and membrane folding, Colloid Surf. A, 183-185(15), 123-132, 2001.

[18] R. Finken and U. Seifert, Wrinkling of microcapsules in shear flow, J. Phys.: Condens. Matter 18, L185-L191, 2006.

[19] V. Kantsler, E. Segre, and V.Steinberg, Vesicle dynamics in time-dependent elongation flow: Wrinkling instability, Phys. Rev. Lett., 99, 178102, 2007.

[20] K.S. Turitsyn and S.S. Vergeles, Wrinkling of vesicles during transient dynamics in elongational flow, Phys. Rev. Lett., 100, 028103, 2008.

[21] P. Sens and S.A. Safran, Pore formation and area exchange in tense membranes, Europhys. Lett., 43(1), 95-100, 1998.

[22] Roy Bar-Ziv, T. Frisch, and E. Moses, Entropic expulsion in vesicles, Phys. Rev. Lett., 75, 3481-3484, 1995.

[23] E. Evans and W. Rawicz, Entropy-driven tension and bending elasticity in condensed-fluid membranes, Phys. Rev. Lett., 64, 2094-2097, 1990.

[24] U. Seifert, The concept of effective tension for fluctuating vesicles, Zeitschrift für Physik B Condensed Matter, 97(2), 2, 299-309, 1995.

[25] S.L. Veatch and S.L. Keller, Separation of liquid phases in giant vesicles of ternary mixtures of phospholipids and cholesterol, Biophys. J., 85(5), 3074-3083, 2003.

[26] T. Baumgart, S. Das, W.W. Webb, and J.T. Jenkins, Membrane elasticity in giant vesicles with fluid phase coexistence, Biophys. J., 89(2), 1067-1080, 2005.

[27] O.Y. Zhong-can and W. Helfrich, Bending energy of vesicle membranes: General expressions for the first, second and third variation of the shape energy and applications to spheres and cylinders, Phys. Rev. A, 39(10), 15, 5280-5288, 1989.

[28] X. Wang and Q. Du, Modelling and simulations of multi-component lipid membranes and open membranes via diffuse interface approaches, J. Math. Biol., 56(3), 347-371, 2008.

[29] J. Xu, Z. Li, J. Lowengrub, and H. Zhao, A level set method for solving interfacial flows with surfactant, J. Comp. Phys., 212, 590-616, 2006.

[30] D. Jamet and C. Misbah, Towards a thermodynamically consistent picture of the phase-field model of vesicles: Local inextensibility, Phys. Rev. E Stat. Nonlin. Soft Matter Phys., 76(5), 1-7, 2007.

[31] D. Jamet and C. Misbah, Thermodynamically consistent picture of the phase-field model of vesicles: Elimination of the surface tension, Phys. Rev. E Stat. Nonlin. Soft Matter Phys., 78, 041903, 2008.

[32] G.K. Bachelor, An Introduction to Fluid Mechanics, Cambridge University Press, New York, 1967.

[33] A.J. James and J. Lowengrub, A surfactant-conserving volume-of-fluid method for interfacial flows with insoluble surfactant, J. Comp. Phys., 201, 685-722, 2004.

[34] L.D. Landau, Statistical Physics, Butterworth-Heinemann, Oxford, 1984.

[35] J. Lowengrub and L. Truskinvosky, Quasi-incompressible cahn-hilliard fluids and topological transitions, Proc. R. Soc. London Ser. A, 454, 2617-2654, 1998.

[36] Z. Li, K. Ito, and M.C. Lai, An augmented approach for stokes equations with a discontinuous viscosity and singular forces, Comp. Fluids, 36, 622-635, 2007.

[37] H. Zhao, T.F. Chan, B. Merriman, and S. Osher, A variational level set approach to multiphase motion, J. Comp. Phys., 127, 179-195, 1996.

[38] J. Xu and H. Zhao, An Eulerian formulation for solving partial differential equations along a moving interface, J. Sci. Comp., 19, 573-594, 2003.

[39] D.J. Eyre, Unconditionally gradient stable time marching in the Cahn-Hilliard equation, in Computational and Mathematical Models of Microstructural Evolution, Mater. Res. Soc. Sympos. Proc., MRS, 529, 39-46, 1998.

[40] P. Smereka, Semi-implicit level set methods for curvature and surface diffusion motion, J. Sci. Comp., 19, 439-456, 2003.

[41] R. LeVeque and $\mathrm{Z}$. Li, The immersed interface method for elliptic equations with discontinuous coefficients and singular sources, SIAM J. Numer. Anal., 31, 1019-1044, 1994.

[42] R. LeVeque and Z. Li, Immersed interface methods for stokes flow with elastic boundaries or surface tension, SIAM. J. Sci. Comp., 18, 709, 1997.

[43] S. Osher and J.A. Sethian, Fronts propagating with curvature dependent speed: Algorithms based on Hamilton-Jacobi formulations, J. Comp. Phys., 79, 12-49, 1988.

[44] S. Osher and R.P. Fedkiw, Level set methods: An overview and some recent results, J. Comp. Phys., 169, 463-502, 2001.

[45] J.A. Sethian and P. Smereka, Level set methods for fluid interfaces, Ann. Rev. Fluid Mech., 
35, 341-372, 2003.

[46] M. Sussman, P. Smereka, and S. Osher, A level set approach for computing solutions to incompressible two-phase flow, J. Comput. Phys., 114, 146-159, 1994.

[47] G.S. Jiang and D. Peng, Weighted eno schemes for Hamilton-Jacobi equations, SIAM J. Sci. Comp., 21, 2126, 2000.

[48] C.W. Shu, Total-variation-diminishing time discretization, SIAM J. Sci. Stat. Comp., 9, 1073$1084,1988$.

[49] V. Cristini, J. Blawzdziewicz, and M. Loewenberg, An adaptive mesh algorithm for evolving surfaces: Simulations of drop breakup and coalescence, J. Comp. Phys., 168, 445-463, 2001.

[50] G.I. Taylor, The formation of emulsions in definable fields of flow, Proc. R. Soc. London A, $146,501-523,1934$

[51] H. Lamb, Hydrodynamics, 6th edition, Cambridge University Press, New York, 1994.

[52] S. Li and J. Sohn, Wrinkling instability of a multicomponent vesicle in viscous fluid, in preparation.

[53] J. Lowengrub, A. Rätz, and A. Voigt, Phase-field modeling of the dynamics of multicomponent vesicles: Spinodal decomposition, coarsening, budding and fission, Phys. Rev. E, 79, 031926, 2009. 